\title{
O-GlcNAcylation of MORC2 at threonine 556 by OGT couples TGF- $\beta$ signaling to breast cancer progression
}

\author{
Ying-Ying Liu ${ }^{1,2,3,4}$, Hong-Yi Liu ${ }^{1}$, Tian-Jian Yu ${ }^{1,2,3,4}$, Qin Lu ${ }^{1}$, Fang-Lin Zhang ${ }^{1,2,3}$, Guang-Yu Liu (D) ${ }^{4}$, Zhi-Ming Shao (iD) ${ }^{1,2,3,4,5}{ }^{\bowtie}$ and \\ Da-Qiang Li (iD) $1,2,3,4,5,6 \bowtie$
}

(c) The Author(s) 2021

MORC family CW-type zinc finger 2 (MORC2) is a newly identified chromatin-remodeling enzyme involved in DNA damage response and gene transcription, and its dysregulation has been linked with Charcot-Marie-Tooth disease, neurodevelopmental disorder, and cancer. Despite its functional importance, how MORC2 is regulated remains enigmatic. Here, we report that MORC2 is O-GlcNAcylated by O-GlcNAc transferase (OGT) at threonine 556. Mutation of this site or pharmacological inhibition of OGT impairs MORC2-mediated breast cancer cell migration and invasion in vitro and lung colonization in vivo. Moreover, transforming growth factor- $\beta 1$ (TGF- $\beta 1$ ) induces MORC2 O-GICNAcylation through enhancing the stability of glutamine-fructose-6-phosphate aminotransferase (GFAT), the rate-limiting enzyme for producing the sugar donor for OGT. O-GlcNAcylated MORC2 is required for transcriptional activation of TGF- $\beta 1$ target genes connective tissue growth factor (CTGF) and snail family transcriptional repressor 1 (SNAIL). In support of these observations, knockdown of GFAT, SNAIL or CTGF compromises TGF- $\beta 1$-induced, MORC2 OGIcNAcylation-mediated breast cancer cell migration and invasion. Clinically, high expression of OGT, MORC2, SNAIL, and CTGF in breast tumors is associated with poor patient prognosis. Collectively, these findings uncover a previously unrecognized mechanistic role for MORC2 O-GICNAcylation in breast cancer progression and provide evidence for targeting MORC2-dependent breast cancer through blocking its O-GIcNAcylation.

Cell Death \& Differentiation (2022) 29:861-873; https://doi.org/10.1038/s41418-021-00901-0

\section{INTRODUCTION}

Cancer cells exert malignant phenotypes partially through reprogramming the posttranslational modification (PTM) patterns of cancer-relevant proteins in response to extracellular and intracellular stimuli [1]. One of such prevalent PTMs is O-linked $\mathrm{N}$-acetylglucosaminylation (O-GlcNAcylation), which is controlled by the concerted actions of a single pair of opposing enzymes, termed O-linked $\mathrm{N}$-acetylglucosamine (O-GlcNAc) transferase (OGT) and O-GlcNAcase (OGA) [2, 3]. OGT transfers a single O-GlcNAc moiety to the hydroxyl group of serine or threonine residues of substrate proteins from the direct donor uridine diphosphate $\mathrm{N}$-acetylglucosamine (UDP-GlcNAc), whereas OGA catalyzes the opposite reaction to cleave O-GIcNAc from O-GlcNAcylated proteins [2, 3].

In addition to OGT and OGA, the cycling of protein O-GlcNAcylation is dependent on the intracellular concentration of UDP-GICNAc, the final product of hexosamine biosynthetic pathway (HBP) [4]. Glutamine-fructose-6-phosphate aminotransferase (GFAT) is the rate-limiting enzyme of HBP, which converts fructose-6-phosphate, an intermediate metabolite in the glycolytic pathway and the first substrate for HBP, to glucosamine-6- phosphate (GlcN-6P). Subsequent acetylation and uridylation of GIcN-6P produce UDP-GIcNAc [4]. Emerging evidence shows that transcription factor CCAAT/enhancer-binding protein B (CEBPB) [5], spliced X-box binding protein 1 (Xbp1s) [6], and multiple extracellular and intracellular signals, such as hypoxia, glucose, glucosamine, nicotine, picolinic acid, and epithelial growth factor [5, 7-9], transcriptionally regulate GFAT expression. In addition, phosphorylation of GFAT by AMP-activated protein kinase (AMPK) [10, 11], calcium/calmodulin-dependent kinase II (CAMK-II) [11], and CAMP-dependent protein kinase (PKA) [12] modulates its enzymic activity. Although GFAT has been documented to be a short-lived protein [13], the underlying mechanisms for controlling its protein turnover remain unknown.

O-GlcNAcylation modification as a signaling moiety alters the functions and activities of target proteins and has extensive crosstalks with other PTMs, such as phosphorylation [3, 4]. Consequently, deregulation of O-GlcNAc cycling has been implicated in the pathogenesis of a plethora of chronic diseases including cancer [4]. In this context, increased levels of O-GlcNAcylation, OGT or GFAT in cancer patients are correlated with cancer progression and a poor prognosis [14]. Pharmacological inhibition

\footnotetext{
${ }^{1}$ Fudan University Shanghai Cancer Center and Shanghai Key Laboratory of Medical Epigenetics, International Co-laboratory of Medical Epigenetics and Metabolism, Ministry of Science and Technology, Institutes of Biomedical Sciences, Fudan University, Shanghai 200032, China. ${ }^{2}$ Cancer Institute, Shanghai Medical College, Fudan University, Shanghai 200032, China. ${ }^{3}$ Department of Oncology, Shanghai Medical College, Fudan University, Shanghai 200032, China. ${ }^{4}$ Department of Breast Surgery, Shanghai Medical College, Fudan University, Shanghai 200032, China. ${ }^{5}$ Shanghai Key Laboratory of Breast Cancer, Shanghai Medical College, Fudan University, Shanghai 200032 , China. ${ }^{6}$ Shanghai Key Laboratory of Radiation Oncology, Shanghai Medical College, Fudan University, Shanghai 200032, China. ${ }^{凶}$ email: liugy688@163.com; zhimingshao@yahoo.com; daqiangli1974@fudan.edu.cn Edited by M. Piacentini
}

Received: 17 February 2021 Revised: 3 November 2021 Accepted: 10 November 2021

Published online: 1 January 2022 
of OGT or GFAT suppresses cancer progression and enhances cellular sensitivity to anticancer agents [15, 16]. Therefore, identification of novel O-GlcNAc-modified substrates not only will lead to a better understanding of the functions of protein $\mathrm{O}$ GlcNAcylation, but also is helpful for the development of new strategies for treating and preventing cancer [4].

Accumulating evidence indicates that chromatin-remodeling proteins have fundamental roles in cancer progression and therapeutic responsiveness through integrating the extracellular and intracellular signals to control gene transcription and DNA damage response [17]. A case in point is MORC family CW-type zinc finger 2 (MORC2), a member of the highly conserved microrchidia (MORC) ATPase protein family [18]. MORC2 protein contains a catalytically active ATPase module, a CW-type zinc finger domain, a chromo-like domain, and four coiled-coil domains [18-20]. We and others recently demonstrated that MORC2 exerts an ATPasedependent chromatin remodeling activity and is involved in DNA damage response [21] and gene transcription [19, 22]. The functional importance of MORC2 in human diseases is highlighted by the fact that its mutations have been linked with Charcot-MarieTooth disease [23-25], neurodevelopmental disorder [26], and cancer [27]. In addition, MORC2 is upregulated in a variety of human tumors and contributes to their aggressive phenotypes $[28,29]$. Recent work from our group shows that MORC2 promotes breast cancer progression and resistance to endocrine therapy and DNA-damaging chemotherapy and radiotherapy [21, 27, 30-32]. Despite its functional importance in human cancer, the regulatory mechanism of MORC2 still remains enigmatic.

In this study, we provide the first evidence that MORC2 is O-GlcNAcylated by OGT at the conserved threonine 556 (T556). Moreover, transforming growth factor- $\beta 1$ (TGF- $\beta 1$ ) induces MORC2 O-GIcNAcylation through enhancing the stability of GFAT. O-GlcNAcylated MORC2 governs the expression of TGF- $\beta 1$ target genes connective tissue growth factor (CTGF) and snail family transcriptional repressor 1 (SNAIL) and is crucial for breast cancer progression. These findings reveal novel mechanistic insights into MORC2-mediated breast cancer progression and provide potential therapeutic opportunities for MORC2-dependent breast cancer through blocking its O-GlcNAcylation.

\section{RESULTS}

\section{OGT interacts with MORC2 in breast cancer cells}

To gain mechanistic insights into the biological functions of MORC2, we recently performed immunoprecipitation (IP) coupled with lipid chromatography-tandem mass spectrometry to characterize MORC2 interacting proteins [32], and identified OGT as a potential binding partner of MORC2 (Supplementary Fig. S1A). To validate this result, HEK293T cells were transfected with FlagMORC2, HA-OGT alone or in combination, and then subjected to reciprocal IP assays with an anti-Flag or anti-HA antibody. Immunoblotting analysis with the indicated antibodies revealed that Flag-MORC2 was co-immunoprecipitated with HA-OGT when both were co-expressed (Fig. 1A, B). Moreover, there was an interaction between MORC 2 and OGT at the endogenous protein level in MCF-7 and T47D cells (Fig. 1C, D). Immunofluorescent staining showed a partial co-localization between MORC2 and OGT in both cell lines (Fig. 1E). Domain-mapping experiments using various deletion constructs further demonstrated that the interaction between MORC2 and OGT was mediated by the $\mathrm{N}$-terminal region of MORC2 (residues 1-420) (Supplementary Fig. S1B, S1C) and the C-terminal domain of OGT (residues 901-1046) (Supplementary Fig. S1D, S1E). These results suggest that OGT interacts with MORC2.

OGT O-GIcNAcylates MORC2 at the conserved threonine $\mathbf{5 5 6}$ As OGT is the unique enzyme for catalyzing O-GlcNAcylation of intracellular proteins [3], we next examined whether MORC2 is modified by O-GlcNAcylation. Toward this aim, HEK293T cells were transfected with Flag-MORC2 and then subjected to IP assays with an anti-Flag antibody. Immunoblotting with a mouse monoclonal anti-O-GlcNAc antibody (RL2) revealed a positive O-GIcNAc signal in the immunoprecipitated MORC2 (Fig. 1F). Moreover, IP assays using an anti-MORC2 antibody revealed that endogenous MORC2 was O-GlcNAcylated in MCF-7 and T47D cells (Fig. 1G). Treatment of MCF-7 and T47D cells with glucose, which produces the donor substrate UDP-GICNAc for OGT through HBP [3], resulted in an increase in MORC2 O-GIcNAcylation in a dose-dependent manner (Fig. $1 \mathrm{H}$ and Supplementary Fig. S1F). These results suggest that MORC2 is an O-GlcNAcylated protein.

We next investigated the involvement of OGT in the noted MORC2 O-GIcNAcylation. As shown in Fig. 11, ectopic expression of wild-type (WT) OGT, but not its catalytically inactive mutant (H558A) [33], enhanced O-GlcNAcylation of exogenously expressed MORC2 in HEK293T cells. Consistently, inhibition of OGT activity by small molecule inhibitor OSMI-1 [34] reduced O-GlcNAcylation levels of exogenous MORC2 in HEK293T cells (Fig. 1J) and of endogenous MORC2 in MCF-7 and T47D cells (Fig. 1K, L). These results were further demonstrated by knockdown of endogenous OGT in MCF-7 and T47D cells using two independent siRNAs (Fig. $1 \mathrm{M}, \mathrm{N}$ ). In vitro O-GlcNAcylation assays using bacterially expressed His-MORC2 and purified HA-OGT protein from HEK293T cells demonstrated that OGT O-GlcNAcylated MORC2 in the presence of UDP-GICNAc (Fig. 10).

To identify the site(s) of MORC2 O-GlcNAcylation, we transiently co-expressed Flag-MORC2 and HA-OGT in HEK293T cells. After immunoprecipitation using anti-Flag $\mathrm{M} 2$ beads and in-gel trypsin digestion, resultant peptides were subjected to electron transfer dissociation-mass spectrometry analysis [35]. By this approach, we identified five potential O-GlcNAcylation sites on MORC2, including T280, T556, T680, S730, and T733 (Supplementary Fig. S2). To determine which residue is the major O-GlcNAcylation site in MORC2, we individually mutated those five residues to alanine (A), which lacks the hydroxy group in the side chain and thus blocks O-GICNAC attachment [36], and then transfected them into HEK293T cells. Sequential IP and immunoblotting analyses with the indicated antibodies showed that mutation of T556, but not the other four residues, resulted in a decrease in MORC2 O-GlcNAcylation levels (Fig. 1P). Moreover, treatment with glucose (Fig. 1Q) or ectopic expression of OGT (Fig. 1R) enhanced the O-GlcNAcylation levels of WT MORC2 in comparison with T556A mutant. These results suggest that T556 is the primary O-GICNAcylation site in MORC2. Sequence alignment revealed that the T556 residue is located within the second coiled-coil domain (residues 547-584) of MORC2 and is highly conserved across species (Fig. 1S). To determine whether potential T556 phosphorylation of MORC2 affects its O-GlcNAcylation, we replaced T556 residue with aspartic acid (T556D) to mimic a constitutively phosphorylated MORC2 at T556. As shown in Fig. 1T, expression of phospho-mimetic T556D mutant or phosphodeficient T556A mutant significantly decreased the levels of MORC2 O-GIcNAcylation. Collectively, these results indicate that T556 is the main O-GlcNAcylation site in MORC2.

\section{OGA de-O-GIcNAcylates MORC2 at T556}

O-GlcNAcylation is a dynamic and reversible process, and the glycosylase catalyzing protein de-O-GlcNAcylation is OGA $[2,3]$. As shown in Fig. $2 A-D$, we demonstrated that MORC2 interacted with OGA at both exogenous and endogenous levels. Moreover, ectopic expression of OGA decreased MORC2 O-GlcNAcylation (Fig. 2E). Conversely, inhibition of OGA activity by small molecule inhibitor PUGNAc [37] enhanced the O-GICNAcylation levels of exogenously expressed MORC2 in HEK293T cells (Fig. 2F) and of endogenous MORC2 in MCF-7 and T47D cells (Fig. 2G, H). Consistently, knockdown of OGA in MCF-7 and T47D cells using two independent siRNAs reduced the O-GlcNAcylation levels of 

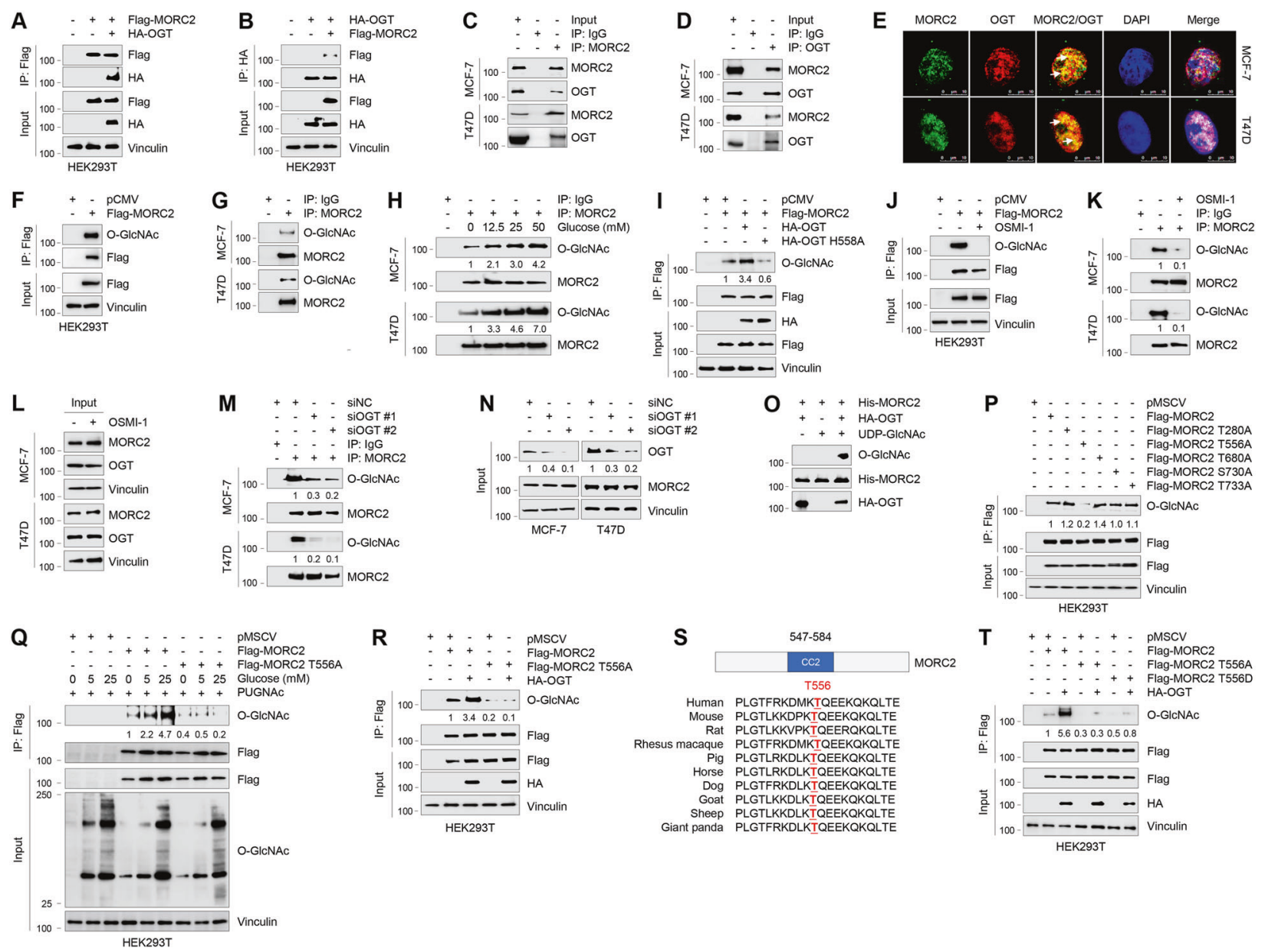

Fig. 1 OGT interacts with MORC2 and O-GIcNAcylates MORC2 at T556. A, B HEK293T cells were transfected with Flag-MORC2 and HA-OGT alone or in combination. IP and immunoblotting analyses were performed with the indicated antibodies after $48 \mathrm{~h}$ of transfection. C, D Lysates from MCF-7 and T47D cells were subjected to IP and immunoblotting analysis with the indicated antibodies. E Immunofluorescence staining of MORC2 and OGT in MCF-7 and T47D cells. Cell nucleus was counterstained with DAPI. F HEK293T cells were transfected with pCMV or FlagMORC2. IP and immunoblotting analyses were performed with the indicated antibodies after $48 \mathrm{~h}$ of transfection. G Lysates from MCF-7 and T47D cells were subjected to IP and immunoblotting analysis with the indicated antibodies. H MCF-7 and T47D cells were cultured in glucoseand serum-free medium for $24 \mathrm{~h}$, and treated with increasing doses of glucose for $24 \mathrm{~h}$. IP and immunoblotting analyses were performed with the indicated antibodies. O-GlcNAc levels were normalized to levels of immunoprecipitated MORC2. The input concerning this experiment is shown in Supplementary Fig. S1F. I HEK293T cells were transfected with Flag-MORC2, HA-OGT or HA-OGT H558A alone or in combination. After $48 \mathrm{~h}$ of transfection, cells were subjected to IP and immunoblotting analysis. O-GlcNAc levels were normalized to levels of immunoprecipitated Flag-MORC2. J HEK293T cells transfected with pCMV or Flag-MORC2 were treated with or without $50 \mu \mathrm{M}$ OSMI-1 for $24 \mathrm{~h}$, and then subjected to IP and immunoblotting analyses with the indicated antibodies. K, L MCF-7 and T47D cells were treated with or without $50 \mu \mathrm{M}$ OSMI- 1 for $24 \mathrm{~h}$ and subjected to IP and immunoblotting analyses with the indicated antibodies. In $\mathrm{K}$, O-GlcNAc levels were normalized to levels of immunoprecipitated MORC2. M, N MCF-7 and T47D cells were transfected with negative control siRNA (siNC) or two independent siRNAs targeting OGT (siOGT). After $48 \mathrm{~h}$ of transfection, cells were subjected to IP and immunoblotting analysis with the indicated antibodies. O-GIcNAc levels were normalized to levels of immunoprecipitated MORC2 (M), and OGT levels were normalized to those of Vinculin (N). 0 HAOGT was purified from HEK293T transfected with HA-OGT. Purified His-MORC2 were incubated with HA-OGT in reaction buffer in a final volume of $25 \mu \mathrm{l}$ per sample. The samples were incubated at $37^{\circ} \mathrm{C}$ for $24 \mathrm{~h}$. MORC2 O-GlcNAc was detected by immunoblotting with an anti-OGlcNAc antibody (RL2). P HEK293T cells were transfected with the indicated expression vectors. After $48 \mathrm{~h}$ of transfection, cells were subjected to IP and immunoblotting analysis with the indicated antibodies. O-GlcNAc levels were normalized to levels of immunoprecipitated FlagMORC2. Q HEK293T cells were transfected with the indicated expression vectors. After $24 \mathrm{~h}$ of transfection, cells were cultured in glucose- and serum-free medium for $24 \mathrm{~h}$ and then treated with increasing doses of glucose for $24 \mathrm{~h}$. IP and immunoblotting analyses were performed with the indicated antibodies. O-GlcNAc levels were normalized to levels of immunoprecipitated Flag-MORC2. R HEK293T cells were transfected with Flag-MORC2 or Flag-MORC2 T556A alone or in combination with HA-OGT. After $48 \mathrm{~h}$ of transfection, cells were subjected to IP and immunoblotting analysis. O-GlcNAc levels were normalized to levels of immunoprecipitated Flag-MORC2. S Alignment of OGT protein sequence among different species. T HEK293T cells were transfected with Flag-MORC2 (WT, T556A, or T556D) alone or in combination with HA-OGT. After $48 \mathrm{~h}$ of transfection, cells were subjected to IP and immunoblotting analysis. O-GlcNAc levels were normalized to levels of immunoprecipitated Flag-MORC2.

endogenous MORC2 (Fig. 2l, J). Moreover, treatment with OGA inhibitor PUGNAc enhanced the O-GlcNAcylation levels of WT MORC2, but not its T556A or T556D mutant (Fig. 2K). Together, these results suggest that OGA de-O-GIcNAcylates MORC2 at T556.
OGT inhibitor OSMI-1 blocks MORC2-mediated breast cancer progression

To address the impact of MORC2 O-GlcNAcylation on biological behavior of breast cancer cells, we first knocked out (KO) endogenous MORC2 in LM2-4175 and BT549 cells using 

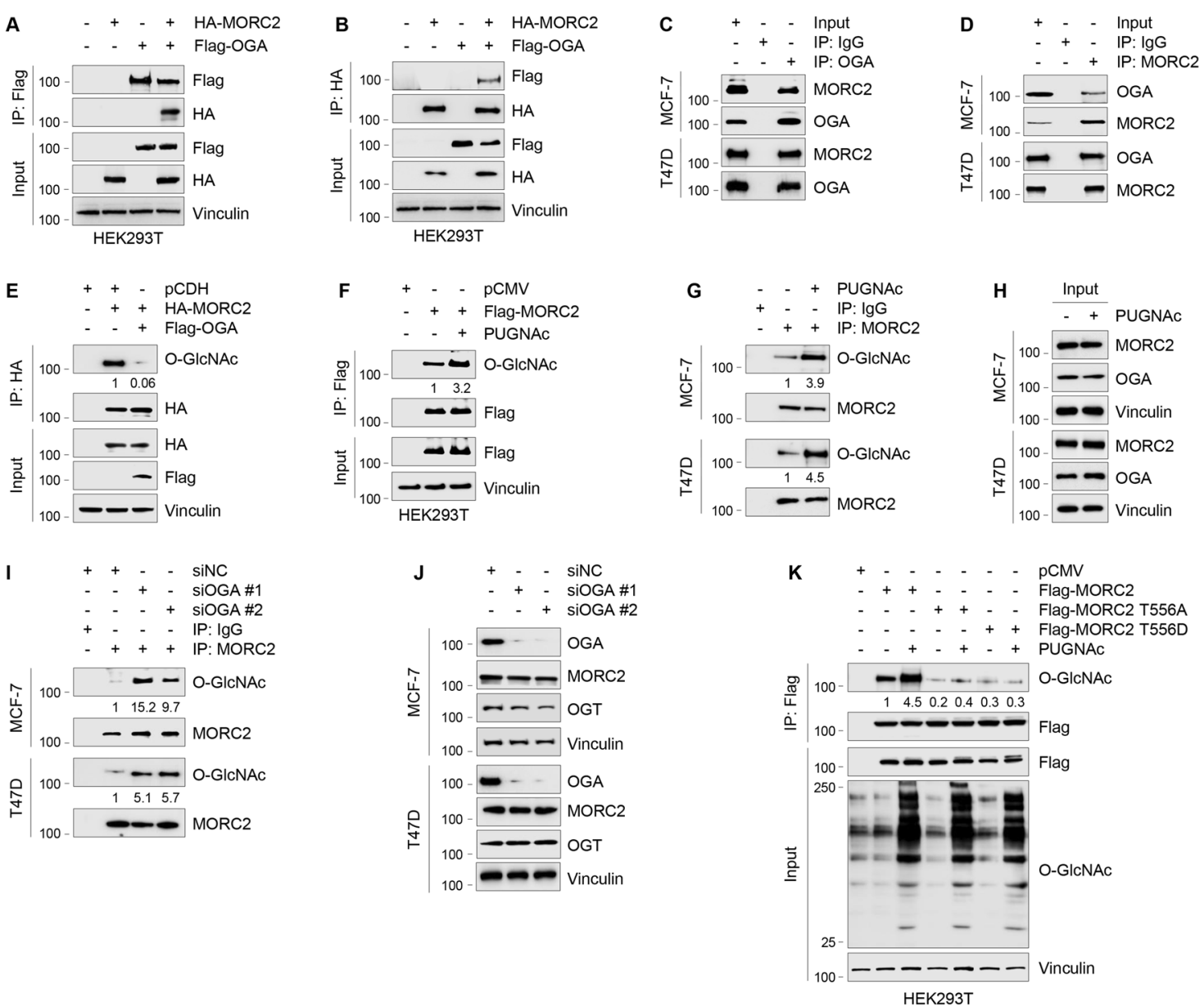

Fig. 2 OGA interacts with MORC2 and de-O-GIcNAcylates MORC2 at T556. A, B HEK293T cells were transfected with HA-MORC2 and FlagOGA alone or in combination. IP and immunoblotting analysis were performed with the indicated antibodies after $48 \mathrm{~h}$ of transfection. C, D Lysates from MCF-7 and T47D cells were subjected to IP and immunoblotting analysis with the indicated antibodies. E HEK293T cells were transfected with Flag-OGA and HA-MORC2 alone or in combination. IP and immunoblotting analysis were performed with the indicated antibodies after $48 \mathrm{~h}$ of transfection. O-GlcNAc levels were normalized to levels of immunoprecipitated HA-MORC2. F HEK293T cells transfected with pCMV or Flag-MORC2 were treated with or without $20 \mu \mathrm{M}$ PUGNAc for $24 \mathrm{~h}$ and subjected to IP and immunoblotting analyses with the indicated antibodies. O-GIcNAc levels were normalized to levels of immunoprecipitated Flag-MORC2. G, H MCF-7 and T47D cells were treated with or without $20 \mu \mathrm{M}$ PUGNAc for $24 \mathrm{~h}$ and subjected to IP and immunoblotting analyses with the indicated antibodies. O-GlcNAc levels were normalized to levels of immunoprecipitated MORC2. I, J MCF-7 and T47D cells were transfected with siNC or two different siRNAs targeting OGA (siOGA). After $48 \mathrm{~h}$ of transfection, cells were subjected to IP and immunoblotting analysis with the indicated antibodies. In I, O-GIcNAc levels were normalized to levels of immunoprecipitated MORC2. K HEK293T cells were transfected with the indicated expression vectors. After $24 \mathrm{~h}$ of transfection, cells were treated with or without $20 \mu \mathrm{M}$ PUGNAc for $24 \mathrm{~h}$ and subjected to IP and immunoblotting analysis. O-GlcNAc levels were normalized to levels of immunoprecipitated Flag-MORC2.

CRISPR-Cas9 technology [38], and then reintroduced empty vector pMSCV or Flag-MORC2 (WT, T556A or T556D) into resultant MORC2 KO cells by lentiviral infection (Fig. 3A). Transwell migration and Matrigel invasion assays showed that knockout of MORC2 reduced the migratory and invasive potential of LM2-4175 and BT549 cells, which was partially restored after reexpression of WT MORC2, but not either T556A (Fig. 3B, C) or T556D (Supplementary Fig. S3) mutant, in MORC2-KO LM2-4175 and BT549 cells. Moreover, treatment with OGT inhibitor OSMI-1 [34] suppressed WT MORC2-mediated breast cancer cell migration and invasion (Fig. 3B, C). In vivo mouse xenograft studies demonstrated that mice injected with cells expressing T556A mutant MORC2 had less metastatic nodules compared to those injected with WT MORC2 expressing cells (Fig. 3D, E). Moreover, administration of OGT inhibitor OSMI-1 suppressed lung metastasis in mice injected with cells expressing WT MORC2 (Fig. 3D, E). Collectively, these results suggest that MORC2 O-GIcNAcylation is critical for breast cancer progression, which can be blocked by OGT inhibitor OSMI-1.

\section{TGF- $\beta 1$ induces MORC2 O-GIcNAcylation at T556 through enhancing GFAT stability}

Protein O-GlcNAcylation is inducible in response to various extracellular or intracellular signals [3]. To investigate the upstream regulatory signals for MORC2 O-GlcNAcylation, we treated MCF-7 cells with three ubiquitous growth factors, including insulin, TGF- $\beta 1$, and epidermal growth factor (EGF), and then determined their impacts on endogenous MORC2 O-GlcNAcylation. Results showed that all of them enabled to induce MORC2 O-GICNAcylation, and TGF- $\beta 1$ showed the strongest response, followed by EGF and insulin (Fig. 4A, B). Furthermore, MORC2 O-GlcNAcylation was enhanced in MCF-7 cells following TGF- $\beta 1$ treatment in a time-dependent manner (Fig. 4C, D). Induction of MORC2 O-GIcNAcylation by TGF- $\beta 1$ was also observed in T47D cells (Supplementary Fig. S4A, S4B). In agreement with these observations, preincubation of TGF- $\beta$ inhibitor SB431542 [39] attenuated TGF- $\beta 1$-induced MORC2 O-GlcNAcylation in MCF-7 (Fig. 4E, F) and T47D (Supplementary Fig. S4C, S4D) cells. Moreover, the O-GlcNAcylation levels of WT 

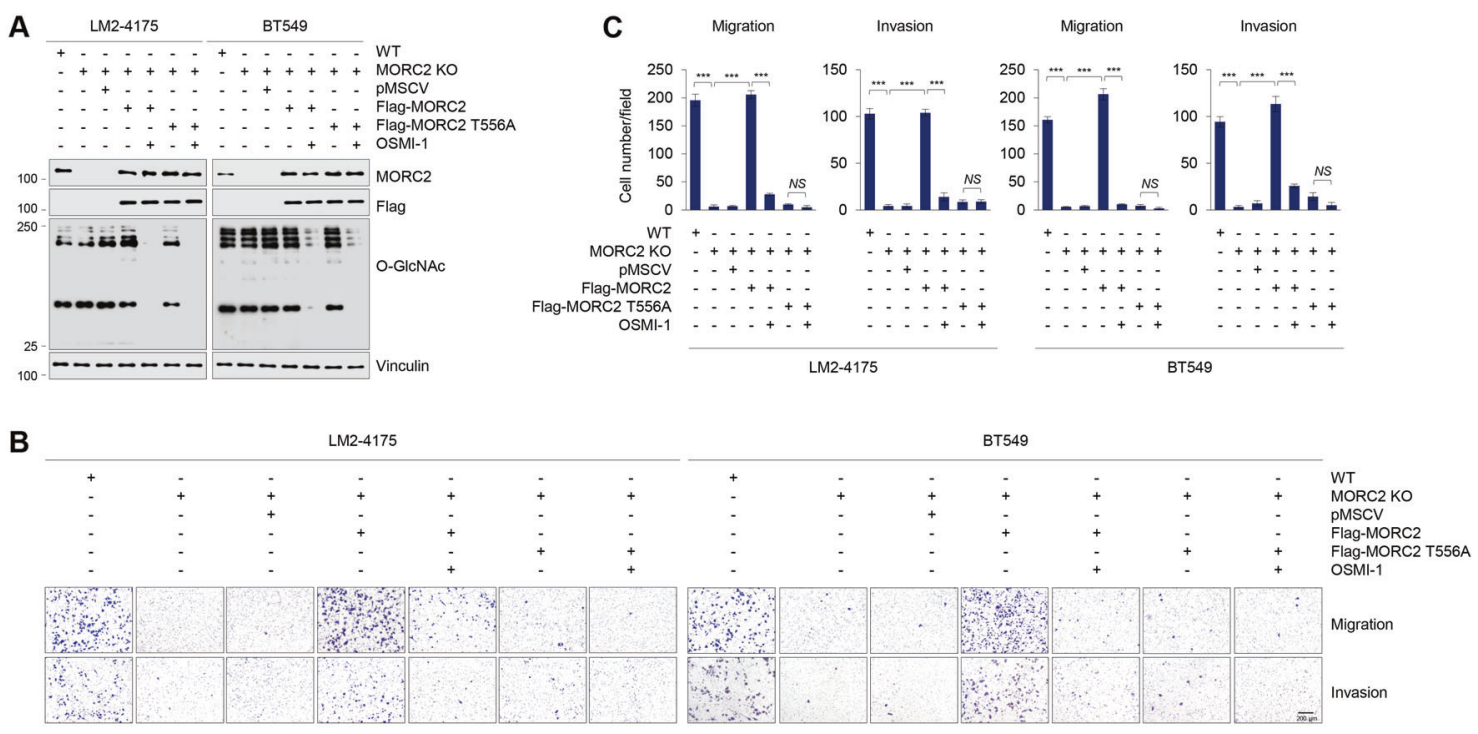

D

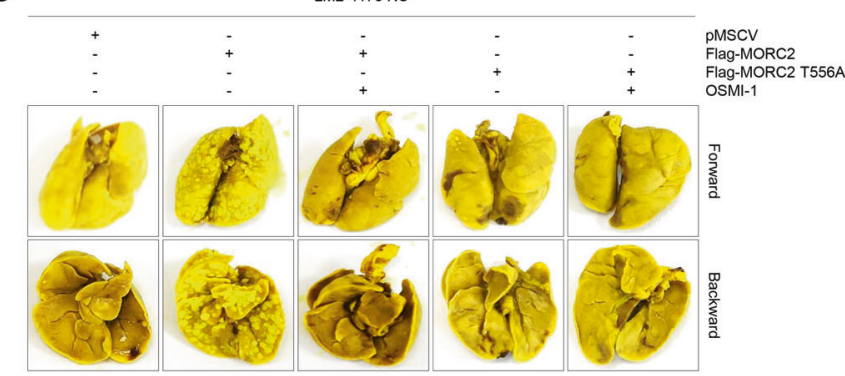

E

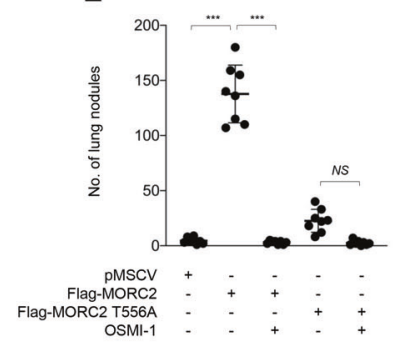

Fig. 3 O-GIcNAcylated MORC2 contributes to breast cancer progression. A-C WT and MORC2-KO LM2-4175 and BT549 cells stably expressing PMSCV, Flag-MORC2 or Flag-MORC2 T556A were treated with or without $50 \mu \mathrm{M}$ OSMI-1 for $24 \mathrm{~h}$, and then subjected to immunoblotting analysis with the indicated antibodies (A) or Transwell migration and invasion assays as described in Materials and Methods. Representative images of migrated and invaded cells (B) and corresponding quantitative results (C) are shown. ***, $p<0.001 ; N S$, no significance. D, E WT and MORC2-KO LM2-4175 and BT549 cells stably expressing pMSCV, Flag-MORC2 or Flag-MORC2 T556A were injected into 6-week-old female BALB/c nude mice $(n=8)$ through the tail vein. OSMI-1 treatment was started 1 week after injection. OSMI-1 was given at a dose of $10 \mathrm{mg} / \mathrm{kg} /$ day for four weeks, five days a week and two days off at weekends. After 5 weeks of injections, the lungs were harvested. Representative images of lung metastasis (D), and quantitative results of lung nodules (E) are shown. ${ }^{* * *} p<0.001 ; N S$, no significance.

MORC2, but not its T556A or T556D mutant, were increased after treatment with TGF- $\beta 1$ (Fig. 4G, H). These results suggest that TGF$\beta 1$ is one of the upstream regulatory signals for MORC2 T556 O-GIcNAcylation.

To investigate the molecular mechanism by which TGF- $\beta 1$ induces MORC2 O-GIcNAcylation, we first examined whether TGF$\beta 1$ affects the interaction of MORC2 with OGT or OGA by IP assays. As shown in Supplementary Fig. S4E-S4G, treatment of MCF-7 and T47D cells with TGF- $\beta 1$ did not significantly affect the binding of MORC2 with OGT or OGA. Interestingly, it was recently documented that treatment of human lung cancer A549 cells with TGF- $\beta 1$ results in an increase in GFAT expression levels [40], but how this occurs remains unexplored. GFAT is the rate-limiting enzyme in HBP for producing the direct donor UDP-GICNAC for OGTmediated protein O-GlcNAcylation [3, 4]. Immunoblotting analyses showed that treatment with TGF- $\beta 1$ resulted in an increase in protein levels of GFAT, but not MORC2, OGT or OGA (Fig. 4l). qPCR analysis showed that the mRNA levels of TGF- $\beta 1$ target gene SNAIL, but not GFAT or MORC2, were upregulated following TGF$\beta 1$ treatment (Supplementary Fig. S5A). These results indicate the effects of TGF- $\beta 1$ on GFAT upregulation to be post-transcriptional.

As GFAT is a short-lived protein [13], we next explored the potential role of the proteasome pathway in controlling its steady- state levels. As shown in Supplementary Fig. S5B, protein levels of GFAT were increased in HEK293T cells after treatment with proteasome inhibitor MG-132. As a control, treatment with MG132 did not significantly affect the protein levels of MORC2, which is known to be degraded through the autophagy-lysosome but not ubiquitin-proteasome pathway [31]. Cycloheximide (CHX) chase assays revealed that TGF- $\beta 1$ treatment enhanced the halflife of GFAT protein (Fig. 4J, K). IP and immunoblotting analysis with the indicated antibodies showed a significant decrease in polyubiquitination levels of GFAT in TGF- $\beta 1$-treated cells (Fig. 4L). These results suggest that TGF- $\beta 1$ stabilizes GFAT by blocking its proteasomal degradation. Furthermore, knockdown of GFAT using two independent siRNAs impaired TGF- $\beta 1$-induced MORC2 O-GlcNAcylation in MCF-7 and T47D cells (Fig. 4M, N).

TGF- $\beta 1$ is frequently overexpressed in human breast tumors and tumor-associated stroma and its upregulation closely correlates with breast cancer cell motility, invasion, and metastasis [41-43]. To address whether MORC2 O-GlcNAcylation is involved in TGF- $\beta 1$-mediated breast cancer cell migration and invasion, we reconstituted empty vector pMSCV or Flag-MORC2 (WT or T556A) into MORC2-KO LM2-4175 and BT549 cells by lentiviral infection and then treated with or without TGF- $\beta 1$ (Supplementary Fig. S5C). Transwell migration and invasion assays showed that treatment 

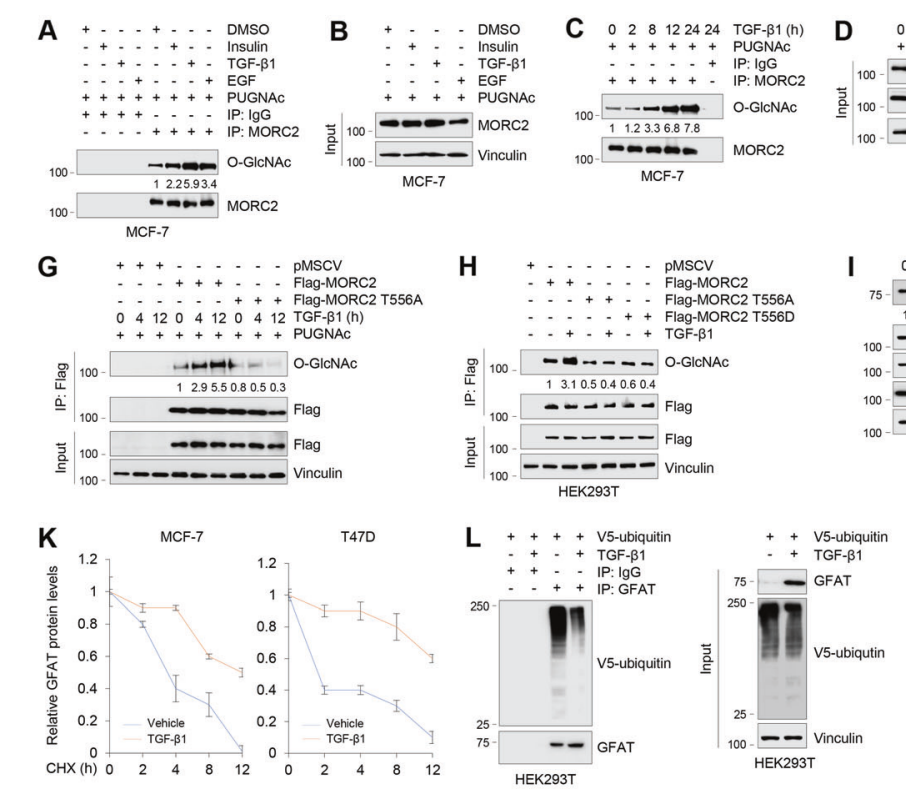
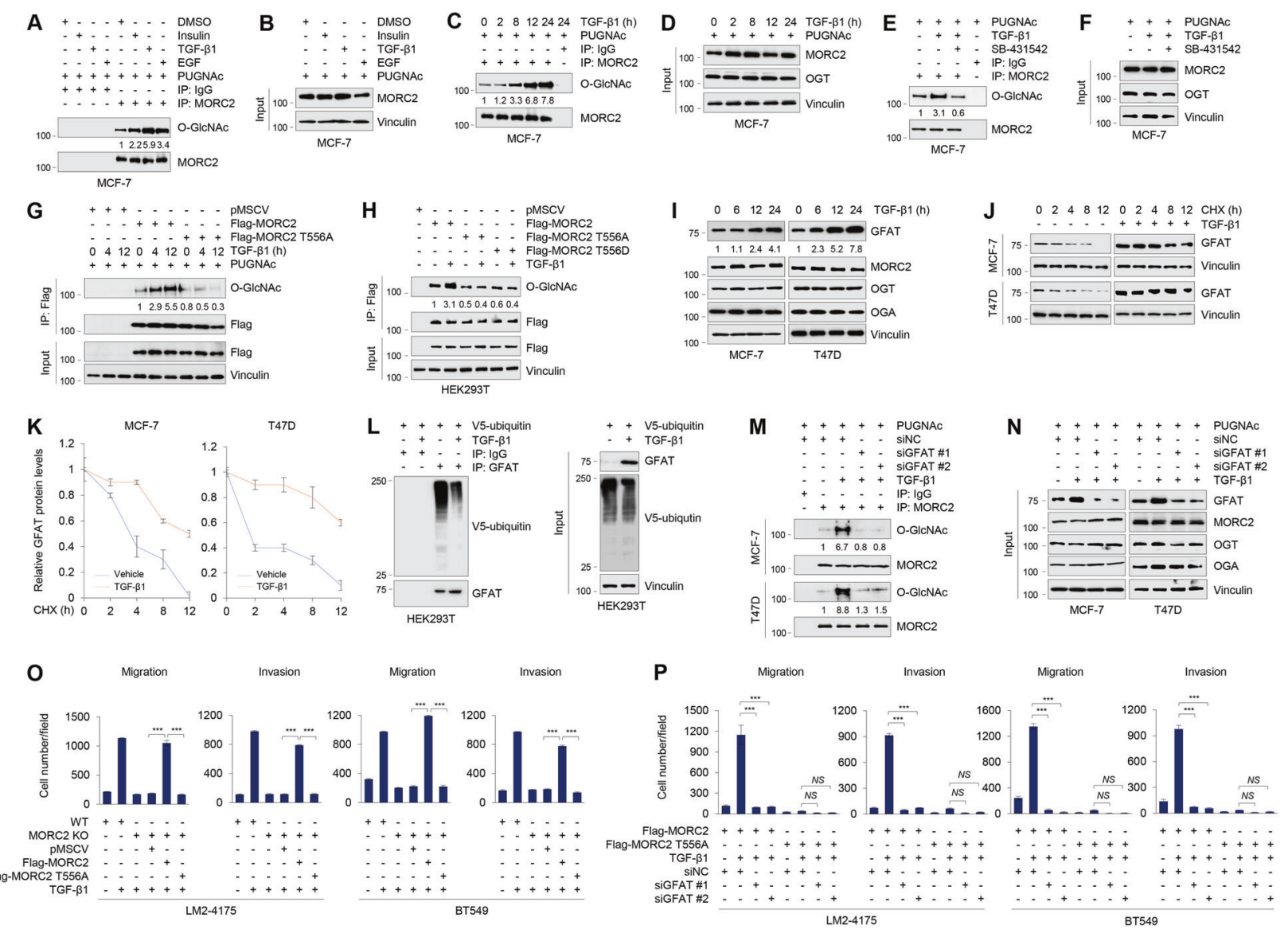

Fig. 4 TGF- $\beta 1$ induces MORC2 O-GIcNAcylation at T556 through enhancing GFAT stability. A, B MCF-7 cells were serum-starved for $24 \mathrm{~h}$ and then treated with or without $10 \mu \mathrm{g} / \mathrm{ml}$ insulin, $5 \mathrm{ng} / \mathrm{ml} \mathrm{TGF-} \beta 1$, or $20 \mathrm{ng} / \mathrm{ml}$ EGF for another $24 \mathrm{~h}$. Cells were harvested for IP and immunoblotting analysis with the indicated antibodies. In A, O-GlcNAc levels were normalized to levels of immunoprecipitated MORC2 levels. C, D MCF-7 cells were serum-starved for $24 \mathrm{~h}$, and then treated with or without $5 \mathrm{ng} / \mathrm{ml} \mathrm{TGF-} \beta 1$ for the indicated times. Cell lysates were subjected to IP and immunoblotting analysis with the indicated antibodies. In C, O-GlcNAc levels were normalized to levels of immunoprecipitated MORC2. E, F MCF-7 cells were serum-starved for $24 \mathrm{~h}$, and then treated with $5 \mathrm{ng} / \mathrm{ml}$ TGF- $\beta 1$ ( $24 \mathrm{~h}$ ) alone or in combination with $5 \mu \mathrm{M}$ TGF- $\beta$ inhibitor SB431542 (12 h). Cells were harvested for IP and immunoblotting analysis with the indicated antibodies. In E, O-GlcNAc levels were normalized to levels of immunoprecipitated MORC2. G, H HEK293T cells were transfected with the indicated expression vectors. After $24 \mathrm{~h}$ of transfection, cells were serum-starved for $24 \mathrm{~h}$, followed by treatment with $5 \mathrm{ng} / \mathrm{ml}$ TGF- $\beta 1 \mathrm{for}$ the indicated times. IP and immunoblotting analysis were performed with the indicated antibodies. In G, O-GlcNAc levels were normalized to levels of immunoprecipitated Flag-MORC2. I MCF-7 and T47D cells were serum-starved for $24 \mathrm{~h}$ and then treated with or without $5 \mathrm{ng} / \mathrm{ml} \mathrm{TGF-}$ $\beta 1$ for the indicated times. Immunoblotting analyses were performed with the indicated antibodies. GFAT levels were normalized to Vinculin levels. J, K MCF-7 and T47D cells were serum-starved for $24 \mathrm{~h}$ and then treated with or without $5 \mathrm{ng} / \mathrm{ml} \mathrm{TGF}-\beta 1$ for $24 \mathrm{~h}$. Cells were treated with $100 \mu \mathrm{g} / \mathrm{ml}$ of cycloheximide (CHX) for the indicated times and then analyzed by immunoblotting (J). Relative expression levels of GFAT to Vinculin are shown in (K). L HEK293T cells were transfected with V5-ubiquitin. After $24 \mathrm{~h}$ of transfection, cells were serum-starved for $24 \mathrm{~h}$ and then treated with or without $5 \mathrm{ng} / \mathrm{ml}$ TGF- $\beta 1$ for another $24 \mathrm{~h}$. IP and immunoblotting analyses were performed with the indicated antibodies. M, N MCF-7 and T47D cells were transfected with siNC or two different siRNAs targeting GFAT (siGFAT). After $24 \mathrm{~h}$ of transfection, cells were serum-starved for $24 \mathrm{~h}$ and then treated with or without $5 \mathrm{ng} / \mathrm{ml}$ TGF- $\beta 1$ for another $24 \mathrm{~h}$. IP and immunoblotting analyses were performed with the indicated antibodies. In M, O-GICNAc levels were normalized to levels of immunoprecipitated MORC2. O WT and MORC2-KO LM2-4175 and BT549 cells stably expressing pMSCV, Flag-MORC2 or Flag-MORC2 T556A were serum-starved for $24 \mathrm{~h}$, treated with or without $5 \mathrm{ng} / \mathrm{ml} \mathrm{TGF}-\beta 1$ for $24 \mathrm{~h}$, and then subjected to Transwell migration and invasion assays. Corresponding quantitative results are shown in O. $*_{* *}^{*}, p<0.001$. Representative images of migrated and invaded cells are shown in Supplementary Fig. S7D. P MORC2-KO LM2-4175 and BT549 cells stably expressing Flag-MORC2 or Flag-MORC2 T556A were transfected with siNC or two siRNAs targeting GFAT (siGFAT). After 24 $\mathrm{h}$ of transfection, cells were serum-starved for $24 \mathrm{~h}$, followed by treatment with or without $5 \mathrm{ng} / \mathrm{ml}$ TGF- $\beta 1$ for $24 \mathrm{~h}$. Transwell migration and invasion assays were performed as described in Materials and Methods. Corresponding quantitative results are shown in $\mathrm{P} .{ }^{* *} p<0.01 .{ }^{* * *}, p<$ 0.001 ; NS, no significance. Representative images of migrated and invaded cells are shown in Supplementary Fig. S7F.

with TGF- $\beta 1$ significantly enhanced the migratory and invasive potential of WT LM2-4175 and BT549 cells relative to MORC2 KO counterparts (Fig. 40 and Supplementary Fig. S5D). Moreover, reconstitution of WT, but not T556A mutant, MORC2, into MORC2KO LM2-4175 and BT549 cells partially restored the responsiveness of MORC2 KO cells to TGF- $\beta 1$ treatment (Fig. 40 and Supplementary Fig. S5D). Moreover, knockdown of GFAT using two independent siRNAs (Supplementary Fig. S5E) impaired TGF$\beta 1$-induced enhancement of migrative and invasive capacity of
MORC2-KO LM2-4175 and BT549 cells re-expressing WT MORC2 (Fig. 4P and Supplementary Fig. S5F). These results suggest that TGF- $\beta 1$ induces O-GlcNAcylation of MORC2 through enhancing the stability of GFAT.

O-GICNAcylated MORC2 regulates CTGF and SNAIL expression As MORC2 and TGF- $\beta$ exert their biological functions through, at least in part, regulating gene transcription [19, 22, 44], we first analyzed two publicly available RNA-sequencing (RNA-Seq) 
datasets, including GSE74377 [45] and GSE95452 [19]. The former includes the mRNA profiles of MCF10A cells with or without TGF$\beta 1$ treatment [45], while the latter contains the expression profiling of WT and MORC2-KO HeLa cells [19]. According to the preset threshold, we found 9 commonly upregulated genes by TGF- $\beta 1$ and MORC2, including SNAIL, CTGF, SPHK1, RHOB, CRYAB, FBN1, PPP2R2B, KIAA1644, and ATP8B2 (Supplementary Fig. S6A). Based on the documented functions of these genes in human cancer (Supplementary Fig. S6B), we selected SNAIL and CTGF for further validation by qPCR assays in MORC2-KO BT549 and MCF-7 cells reconstituted with empty vector pMSCV, WT or T556A mutant MORC2. Results showed that treatment with TGF- $\beta 1$ (Fig. 5A, B), OGA inhibitor PUGNAc (Fig. 5C, D) or ectopic expression of HAOGT (Fig. 5E, F) resulted in an upregulation in CTGF and SNAIL mRNA levels in MORC2-KO BT549 and MCF-7 cells re-expressing WT MORC2 relative to those expressing T556A or T556D mutant MORC2. These results were further demonstrated by immunoblotting assays (Fig. 5G-I). The results indicate that MORC2 T556 O-GlcNAcylation transcriptionally activates CTGF and SNAIL expression.

As SNAIL and CTGF positively regulate each other [46-49], we then focused on addressing the underlying mechanism by which MORC2 T556 O-GlcNAcylation regulates SNAIL and CTGF expression. To examine the recruitment of MORC2 onto SNAIL and CTGF promoter, we treated MORC2-KO BT549 and MCF-7 cells reexpressing empty vector pMSCV and Flag-MORC2 with or without TGF- $\beta 1$, and then immunoprecipitated crosslinked chromatin with an anti-Flag antibody or IgG as a control. qPCR assays were then performed using primers designed against about every $500 \mathrm{bp}$ region of SNAIL and CTGF promoter (Supplementary Fig. S7A, S7B). Results showed that MORC2 was recruited to one region (R4) of SNAIL promoter ( -151 to -476$)$ and two regions (R3 and R4) of CTGF promoter ( -1035 to -1235 and -1534 to -1925$)$, and these events were enhanced following TGF- $\beta 1$ treatment (Fig. $6 \mathrm{~A}$ and Supplementary Fig. S7C). Furthermore, mutation of O-GlcNAcylation site in MORC2 (T556A) reduced its recruitment to SNAIL promoter (R4) and CTGF promoter (R3 and R4), and this phenomenon was not significantly affected by TGF- $\beta 1$ treatment (Fig. 6B and Supplementary Fig. S7D). To examine whether CTGF and SNAIL promoter activities are affected by MORC2 O-GlcNAcylation, we generated two luciferase reporter constructs containing R4 of SNAIL promoter ( $p$ GL3-SNAIL +100 to -500 ) and R3 and R4 of CTGF promoter (pGL3-CTGF -901 to -2000 ), and then transfected them into MORC2-KO BT549 and MCF-7 cells reconstituted with WT or T556A mutant MORC2. Luciferase reporter assays revealed that treatment with TGF- $\beta 1$ (Fig. 6C, F), PUGNAC (Fig. 6D, G), or ectopic expression of OGT (Fig. $6 \mathrm{E}, \mathrm{H}$ ) enhanced the promoter activities of SNAIL and CTGF in MORC2-KO BT549 and MCF-7 cells re-expressing WT MORC2 relative to its T556A mutant. These results suggest that MORC2 O-GlcNAcylation could affect SNAIL and CTGF promoter activities. Moreover, knockdown of endogenous SNAIL or CTGF using two independent siRNAs (Supplementary Fig. S8A, S8B) impaired TGF$\beta 1$-induced enhancement of migrative and invasive capacity of MORC2-KO LM2-4175 and BT549 cells re-expressing WT MORC2

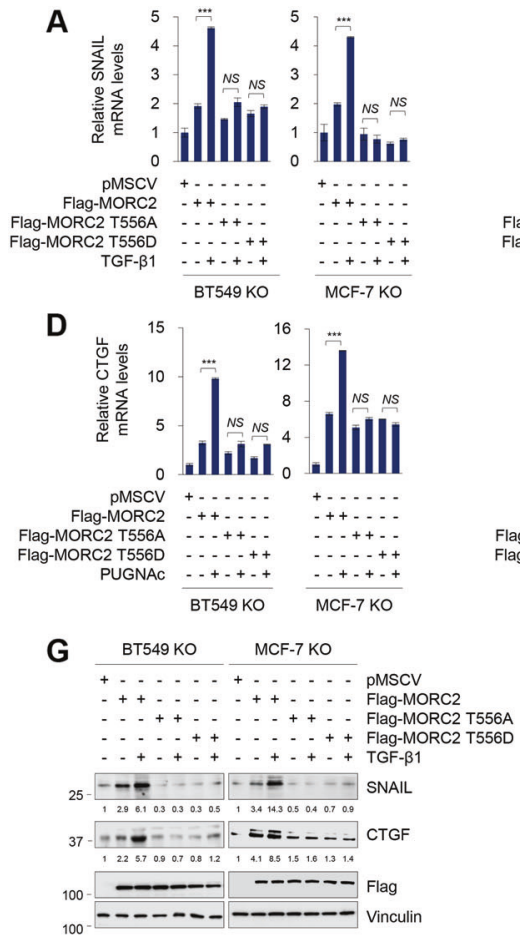

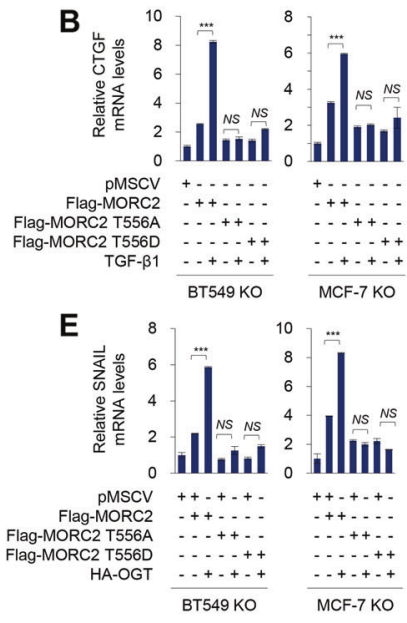

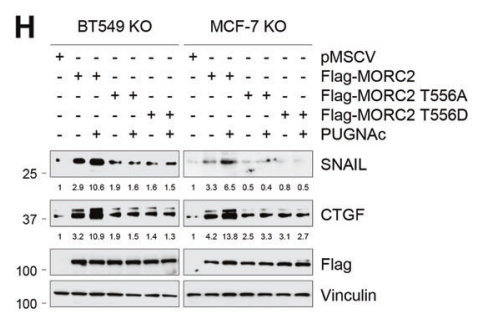

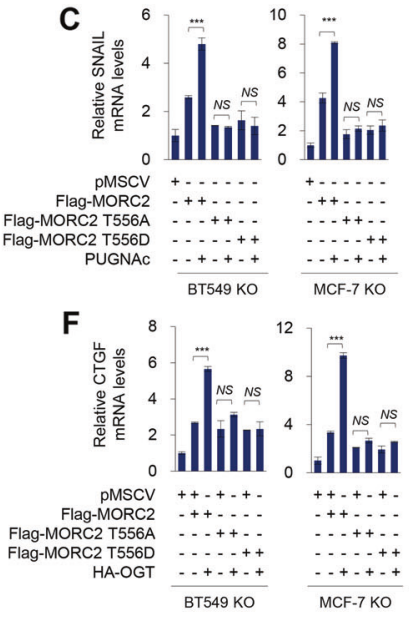

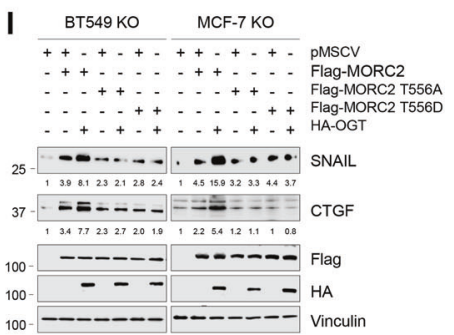

Fig. 5 O-GICNAcylated MORC2 transcriptionally regulates CTGF and SNAIL expression. A, B MORC2-KO MCF-7 and BT549 cells stably expressing pMSCV or Flag-MORC2 (WT, T556A, or T556D) were serum-starved for $24 \mathrm{~h}$, treated with or without $5 \mathrm{ng} / \mathrm{ml}$ TGF- $\beta 1 \mathrm{for}$ another 24 $\mathrm{h}$, and then subjected to qPCR analysis of SNAIL (A) or CTGF (B) mRNA levels. ${ }^{* *}, p<0.001$; NS, no significance. C, D MORC2-KO MCF-7 and BT549 cells stably expressing pMSCV or Flag-MORC2 (WT, T556A, or T556D) were treated with or without $20 \mu \mathrm{M}$ PUGNAc for $24 \mathrm{~h}$ and then subjected to qPCR analysis of SNAIL (C) or CTGF (D) mRNA levels. ${ }^{* * *}, p<0.001$; NS, no significance. E, F MORC2-KO MCF-7 and BT549 cells stably expressing pMSCV or Flag-MORC2 (WT, T556A, or T556D) were transfected with or without HA-OGT. After $48 \mathrm{~h}$ of transfection, cells were subjected to qPCR analysis of SNAIL (E) or CTGF (F) mRNA levels. ${ }^{* * *}, p<0.001$. NS, no significance. G, H MORC2-KO MCF-7 and BT549 cells stably expressing pMSCV or Flag-MORC2 (WT, T556A or T556D) were treated with or without $5 \mathrm{ng} / \mathrm{ml}$ TGF- $\beta 1$ for $24 \mathrm{~h}$ (G) or $20 \mu \mathrm{M}$ PUGNAC for $24 \mathrm{~h}(\mathrm{H})$, then and subjected to immunoblotting analysis. CTGF and SNAIL levels were normalized to Vinculin levels. In G, cells were serumstarved for $24 \mathrm{~h}$ prior to TGF- $\beta 1$ treatment. I MORC2-KO MCF-7 and BT549 cells stably expressing pMSCV or Flag-MORC2 (WT, T556A or T556D) were transfected with or without HA-OGT. After $48 \mathrm{~h}$ of transfection, cells were subjected to immunoblotting analysis. CTGF and SNAIL levels were normalized to Vinculin levels. 

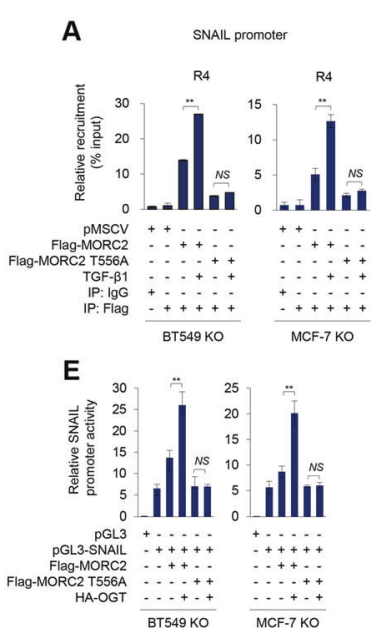

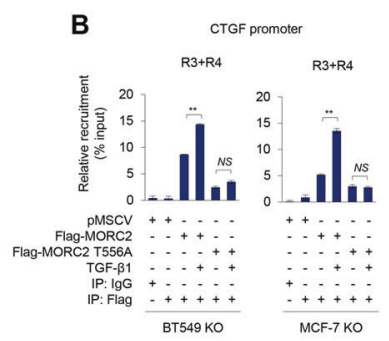

$\mathbf{F}$

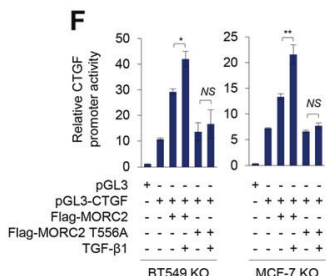

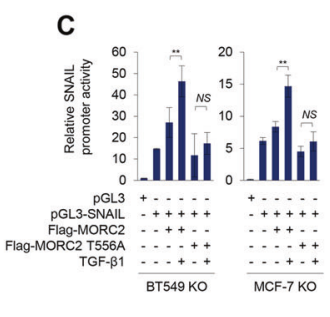

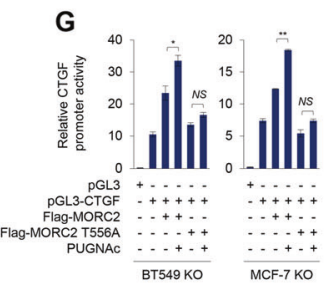

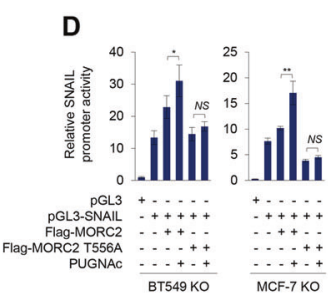

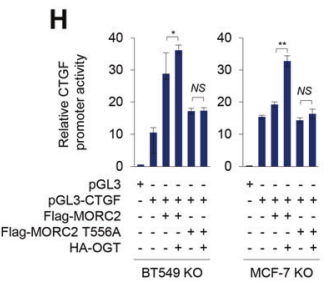

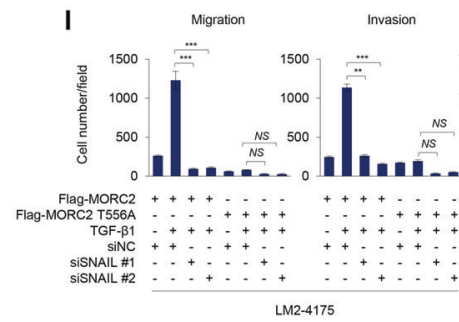
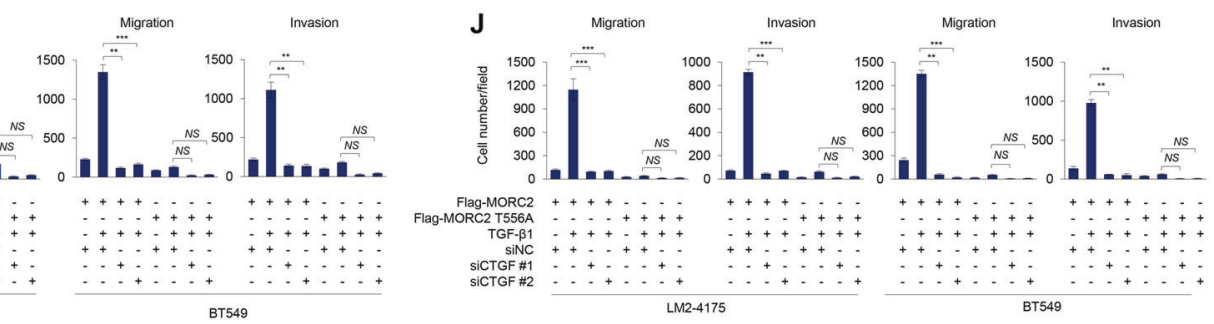

Fig. 6 O-GlcNAcylated MORC2 is recruited to SNAIL and CTGF promoters and enhances promoter activities of SNAIL and CTGF. A, B MORC2-KO BT549 and MCF-7 cells stably expressing pMSCV or Flag-MORC2 (WT or T556A) were serum-starved for $24 \mathrm{~h}$, and then treated with or without $5 \mathrm{ng} / \mathrm{ml} \mathrm{TGF-} \beta 1$ for $24 \mathrm{~h}$. ChIP assays were performed with an anti-Flag antibody or IgG, followed by qPCR analysis. Recruitment of Flag-MORC2 to the SNAIL (A) or CTGF (B) promoter was normalized to Input. ${ }^{* *} p<0.01$; NS, no significance. C, D, F, G MORC2-KO MCF-7 and BT549 cells stably expressing PMSCV or Flag-MORC2 (WT or T556A) were transfected with a luciferase reporter construct encoding pGL3, pGL3-CTGF ( -901 to -2000$)$ or pGL3-SNAIL ( +100 to -500$)$. After $24 \mathrm{~h}$ of transfection, cells were treated with or without $5 \mathrm{ng} / \mathrm{ml}$ TGF$\beta 1$ for $24 \mathrm{~h}$ (C and F) or $20 \mu \mathrm{M}$ PUGNAc for $24 \mathrm{~h}$ (D and G). In D and G, cells were serum-starved for $24 \mathrm{~h}$ prior to TGF- $\beta 1$ treatment. Luciferase assays were performed as described in Materials and Methods. Transfection efficiency was normalized to co-transfected Renilla (Ren) luciferase. Results represent three independent experiments. Error bars represent SEM. ${ }^{* *} p<0.01$; NS, no significance. E, H MORC2-KO MCF-7 and BT549 cells stably expressing pMSCV or Flag-MORC2 (WT or T556A) were co-transfected with or without a luciferase reporter construct encoding pGL3, pGL3-SNAIL ( +100 to -500$)$ (E) or pGL3-CTGF ( -901 to -2000$)(\mathrm{H})$ and HA-OGT. After $48 \mathrm{~h}$ of transfection, luciferase assays were performed as described above. The results are representative of three independent transfection experiments. Error bars represent SEM. ${ }^{* *} p<0.01 ; N S$, no significance. I, J MORC2-KO LM2-4175 and BT549 cells stably expressing Flag-MORC2 or Flag-MORC2 T556A were transfected with siNC or two siRNAs targeting SNAIL (siSNAIL) (Supplementary Fig. S10A) or CTGF (siCTGF) (Supplementary Fig. S10B). After 24 $\mathrm{h}$ of transfection, cells were serum-starved for $24 \mathrm{~h}$, followed by treatment with or without $5 \mathrm{ng} / \mathrm{ml} \mathrm{TGF}-\beta 1$ for $24 \mathrm{~h}$. Transwell migration and invasion assays were performed as described in Materials and Methods. Corresponding quantitative results are shown in I-J. ${ }^{* *} p<0.01 .{ }^{* * *}$, $p<0.001 ; N S$, no significance. Representative images of migrated and invaded cells are shown in Supplementary Fig. S10C and Fig. S10D.

(Fig. $6 \mathrm{I}$, J and Supplementary Fig. S8C, S8D). Together, these results suggest that GFAT-MORC2 O-GICNAcylation-SNAIL/CTGF pathway is involved in TGF- $\beta 1$-induced breast cancer cell migration and invasion.

\section{High expression of OGT, MORC2, SNAIL, and CTGF in breast tumors is correlated with poor patient prognosis}

To determine the clinical relevance of our findings, we performed immunohistochemical (IHC) staining using a tissue microarray (TMA) containing 126 human breast tumor samples with an antibody against MORC2, O-GICNAC, OGT, GFAT, SNAIL, or CTGF. Characterization of clincopathological features of these patients is shown in Supplementary Table S1. Representative IHC images of staining intensity of those six proteins are shown in Fig. 7A. Quantitative results showed that there was a positive correlation in expression levels between OGT and O-GlcNAcylation (Fig. 7B), GFAT and CTGF (Fig. 7C), GFAT and SNAIL (Fig. 7D), MORC2, OGT, and CTGF, or MORC2, OGT and SNAIL (Fig. 7E) in these samples. Due to the well-recognized technical difficulty in generating O-GIcNAc site-specific antibodies at present [50], we utilized the co-expression status of MORC2 and OGT to indirectly reflect the levels of MORC2 T556 O-GlcNAcylation. The Kaplan-Meier method and log-rank test showed that patients whose tumors had high expression of both MORC2 and OGT proteins had poorer overall survival (OS) and disease-free survival (DFS) than those with low expression of MORC2 and OGT (Fig. 7F, G). Similarly, OS and DFS of the patients with high expression of all of three proteins (MORC2, OGT, and CTGF or MORC2, OGT, and SNAIL) were shorter than those of patients with low proteins expression of all of three proteins (Fig. $7 \mathrm{H}-\mathrm{K}$ ). Taken together, these results support the functional role of OGT-MORC2 O-GIcNAcylation-CTGF/SNAIL axis in clinical breast cancer progression.

\section{DISCUSSION}

In this study, we uncovered several interesting findings concerning the functional and mechanistic role of OGT-mediated MORC2 O-GlcNAcylation in breast cancer progression (Supplementary Fig. S9).

First, O-GlcNAcylation is a novel PTM of MORC2, which is required for breast cancer progression. MORC2 is an emerging oncoprotein that is upregulated in various types of human cancer and contributes to cancer cell growth, metastasis, and chemoresistance [27-29, 51]. We recently demonstrated that MORC2 undergoes signaling-dependent phosphorylation [31], acetylation [30], and poly(ADP-ribosyl)ation [32], and these PTMs are 

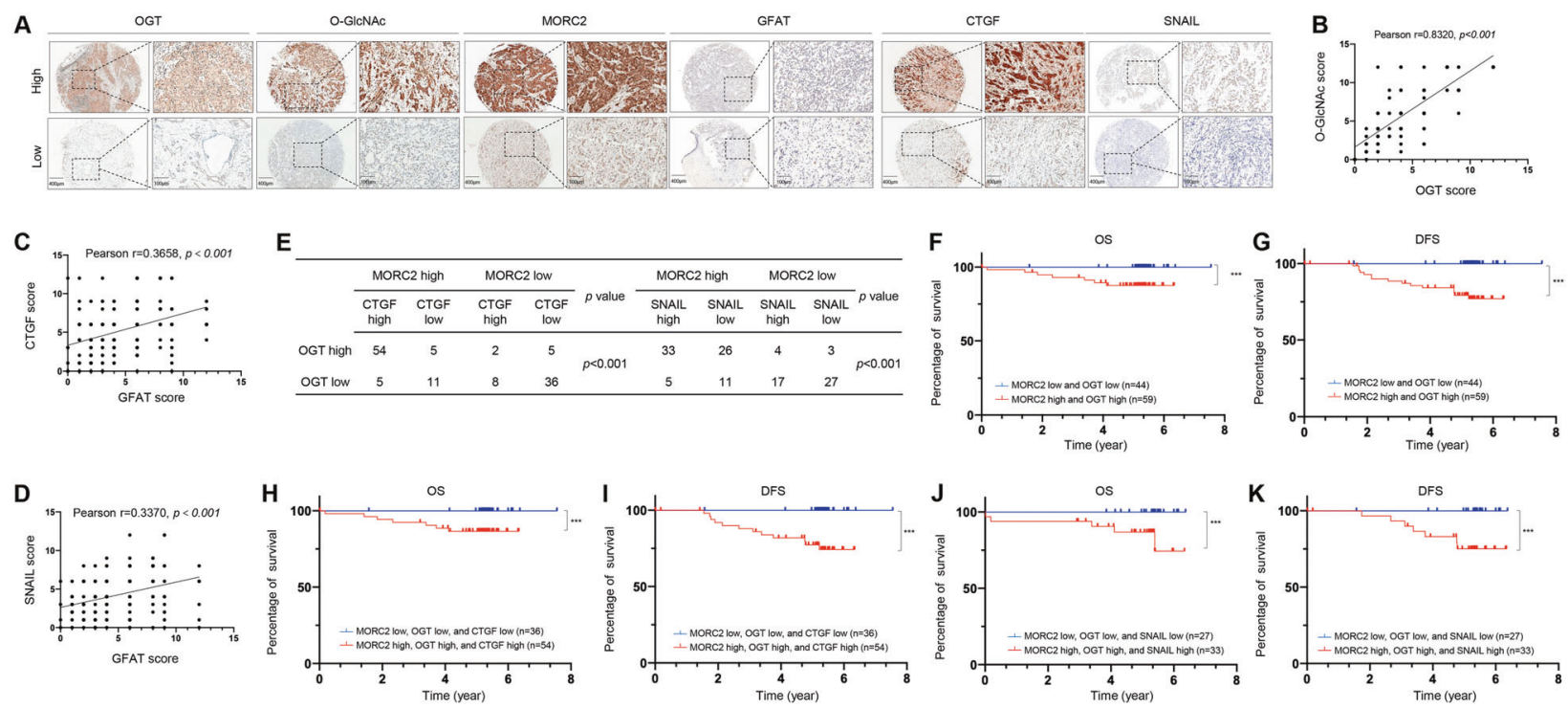

Fig. 7 High expression of MORC2, OGT, and SNAIL/CTGF in breast tumors is associated with poor patient prognosis. A IHC staining was carried out on a tissue microarray containing 126 breast tumor samples with clinical fellow-up information. Representative IHC images of MORC2, OGT, GFAT, SNAIL, CTGF, and O-GIcNAc expression are shown. Scale bars, $400 \mu \mathrm{m}$ (left without magnification) and $100 \mu \mathrm{m}$ (right with magnification). B-D Correction analysis of OGT and O-GlcNAc (B), GFAT and CTGF (C), GFAT, and SNAIL (D) expression levels in 126 breast tumor tissues. Pearson correlation test was used. E Correction analysis of MORC2/OGT/CTGF and MORC2/OGT/SNAIL expression levels in 126 breast tumor tissues. Pearson correlation test was used. F, G Kaplan-Meier curves of OS (F) and DFS (G) of 126 breast cancer patients with high or low expression of both MORC2 and OGT proteins are shown. ${ }^{* * *}, p<0.001$. H, I Kaplan-Meier curves of OS (H) and DFS (I) of 126 breast cancer patients with high or low expression of all of three proteins (MORC2, OGT, and CTGF). J, K Kaplan-Meier curves of OS (J) and DFS (K) of 126 breast cancer patients with high or low expression of all three proteins (MORC2, OGT, and SNAIL) are shown. ${ }^{* * *}, p<0.001$.

implicated in the resistance of breast cancer cells to endocrine therapy drugs and DNA-damaging therapeutic agents. In this study, we provide the first evidence that MORC2 is subjected to O-GlcNAcylation modification at T556, and this process is regulated by OGT and OGA (Figs. 1 and 2). These results add another layer of complexity to the PTM code in regulating biological functions of MORC2. Although many proteins are known to undergo O-GlcNAcylation, only a few site-specific functions for protein O-GlcNAcylation have been reported, including Tau (S400) [52], SIRT1 (S549) [53], TAB1 (S395) [54], c-Myc (T58) [55], and IRS-1 (S1011) [56]. In this study, we showed that mutation of MORC2 O-GlcNAcylation site (T556A) or pharmacological inhibition of OGT by OSMI-1 impairs MORC2mediated breast cancer cell migration and invasion in vitro and lung colonization in a mouse xenograft mouse model (Fig. 3). These results suggest that MORC2 O-GlcNAcylation contributes to breast cancer progression, which is blocked by OGT inhibitor OSMI-1.

Second, TGF- $\beta 1$ induces MORC2 O-GlcNAcylation by enhancing the stability of GFAT. HBP controls the production of UDPGICNAC, the donor substrate for OGT, and the first and limiting step of HBP is catalyzed by GFAT $[4,57]$. The expression levels of GFAT are elevated in multiple types of human cancer and are associated with a poor patient prognosis [58-63]. Depletion or pharmacological inhibition of GFAT suppresses tumor growth and sensitizes cancer cells to anti-PD1 therapy and cisplatin cytotoxicity [15, 60, 64]. GFAT has been shown to be transcriptionally regulated [5-9], and its enzymatic activities are modulated by phosphorylation [10-12]. In this study, we demonstrated that GFAT is subjected to proteasomal degradation, and TGF- $\beta 1$ enhances the stability of GFAT through reducing its ubiquitination (Fig. 4). Furthermore, TGF- $\beta 1$ enhances MORC2 O-GIcNAcylation, which is dependent on GFAT (Fig. 4). However, how TGF- $\beta 1$ regulates the ubiquitination of GFAT remains to be addressed in the future. In agreement with our results, it has been shown that cytokine interleukin-1 induces $\mathrm{O}-$-GIcNAcylation of TAB1 to modulate TGF- $\beta$-activated kinase 1 (TAK1)-mediated cytokine release [54]. In addition, previous studies have shown that overexpression of GFAT induces TGF- $\beta 1$ synthesis in NIH-3T3 fibroblasts [65], and GFAT enzyme activity is necessary for the induction of TGF- $\beta 1$ expression in mesangial cells [66]. Thus, whether there is a positive feedback loop between GFAT and TGF- $\beta 1$ to induce MORC2 O-GlcNAcylation in breast cancer cells remains to be addressed in the future.

Third, O-GlcNAcylated MORC2 transcriptionally activates TGF- $\beta 1$ target genes CTGF and SNAIL. TGF- $\beta$ signaling contributes to breast cancer progression through, at least in part, transcriptional regulation of tumor metastasis-related genes, such as SNAIL [67] and CTGF [68]. SNAIL is a master regulator of TGF- $\beta$-driven epithelial-mesenchymal transition and a strong inducer of breast cancer invasion and metastasis [69-72]. Similarly, CTGF plays a crucial role in the migratory, invasive, metastatic, and angiogenic processes in human breast cancer cells [73, 74]. Moreover, elevated levels of CTGF in primary breast tumors are associated with more advanced features [75]. Through analysis of publicly available RNA-seq databases followed by validation by immunoblotting and qPCR assays, we found that SNAIL and CTGF are transcriptionally regulated by TGF- $\beta 1$-indcued MORC2 O-GlcNAcylation (Fig. 5). O-GlcNAcylated MORC2 is recruited to SNAIL and CTGF promoter and enhances their activities (Fig. 6 and Supplementary Fig. S7). Furthermore, expression of an OGIcNAcylation-defective MORC2 or knockdown of CTGF or SNAIL impairs TGF- $\beta 1$-induced breast cancer cell migration and invasion (Fig. 6 and Supplementary Fig. S8). These results indicate that TGF$\beta$-MORC2 O-GICNAcylation-CTGF/SNAIL signaling axis is implicated in breast cancer progression.

In conclusion, the findings presented here uncover a previously unrecognized functional and mechanistic role for MORC2 O-GIcNAcylation in breast cancer progression. OSMI-1, an OGT inhibitor, significantly suppresses MORC2-mediated breast cancer cell invasion and metastasis. Therefore, targeting O-GIcNAc signaling may be a potential therapeutic approach for MORC2mediated breast cancer progression. 


\section{MATERIALS AND METHODS}

\section{Cell cultures and treatment}

Human breast cancer cell lines (MCF-7, T47D, and BT549) and human embryonic kidney 293 T (HEK293T) cell line were obtained from the Cell Bank of the Chinese Academy of Sciences (Shanghai, China). MDA-MB-231derived LM2-4175 cells were kindly provided by Guohong Hu (University of Chinese Academy of Sciences, Shanghai, China). All cell lines were authenticated by detection of mycoplasma, DNA-fingerprinting, isozyme, and cell vitality. Cell lines were expanded and frozen immediately into numerous aliquots after arrival. The cells revived from the frozen stock were used within 10-15 passages and a period of 6 months.

Cells were maintained in DMEM (BasalMedia, \#L110) media supplemented with $10 \%$ fetal bovine serum (ExCell Bio, \#FSP500) and 1xpenicillinstreptomycin solution (BasalMedia, \#S110B). DMEM media without glucose (Thermo Fisher, \#11966025) was used for glucose-starved experiments. For glucose treatment, cells were cultured in glucose- and serum-free medium for $24 \mathrm{~h}$ and then incubated with glucose at indicated concentrations for another $24 \mathrm{~h}$. For TGF- $\beta 1$ treatment, cells were serum-starved for $24 \mathrm{~h}$ and then incubated with or without $5 \mathrm{ng} / \mathrm{ml}$ recombinant human TGF- $\beta 1$ (Cell Signaling Technology, \#8915LF) for the indicated times. Unless otherwise stated, all reagents were purchased from Sigma-Aldrich. The detailed information for chemical reagents is provided in Supplementary Table S2.

\section{Expression vectors, plasmid transfection, and lentiviral infection}

Myc-DDK-tagged MORC2 was obtained from Origene (\#RC200518), and then subcloned into the lentiviral vector PCDH-CMV-MCS-EF1-Puro (System Biosciences, \#CD510B-1) or pMSCV-Hyg to generate HA-MORC2 and FlagMORC2. cDNAs for OGT (\#CH806183) and OGA (\#CH801095) in pEnter vector were obtained from Vigene Biosciences. CDNA for OGT was subcloned into the lentiviral vector pCDH-CMV-MCS-EF1-Puro (System Biosciences, \#CD510B-1) to generate HA-OGT using CloneEZ PCR Cloning Kit (Genscript, \#L00339). Site-directed mutagenesis was generated by conventional PCR-based method. Expression vector encoding V5-ubiquitin has been described previously [32]. Generation of MORC2 knockout (KO) cells using single-guide RNA sequences (sgRNA) targeting MORC2 by CRISPR/Cas9 technology has been described previously [27, 30, 32]. The CTGF and SNAI promoter regions ( -900 to -2000 and +100 to -500 ) were synthesized by GENEWIZ Biotech and cloned into a pGL3-basic luciferase reporter vector (Promega, \#E1751). All construct sequences were verified by DNA sequencing. The detailed information concerning expression constructs and the primers used for molecular cloning is provided in Supplementary Tables S3 and S4. Transient plasmid transfection was performed using Neofect DNA transfection reagent (TengyiBio, \#TF201201) according to the manufacturer's protocol. Lentiviral infection and generation of stable cell lines were carried out as described previously [27, 30, 32].

\section{Small interfering RNAs (siRNAs) and transfection}

Specific siRNAs targeting OGT, OGA, GFAT, SNAIL, CTGF, and corresponding negative control siRNAs (siNC) were purchased from GenePharma (Shanghai, China), and their targeting sequences are listed in Supplementary Table S5. The siRNA duplexes were transfected into cells using Lipofectamine 2000 transfection reagents (Invitrogen, \#11668019) following the manufacturer's instructions. Knockdown efficiency was determined by immunoblotting analysis after $48 \mathrm{~h}$ of transfection.

\section{Antibodies, immunoblotting, immunoprecipitation assays}

All primary and secondary antibodies used in this study are summarized in Supplementary Table S6. Immunoblotting analysis and IP assays were performed as described previously [27, 30,32]. The optical density of immunoblotting bands was quantified using ImageJ program and was normalized to the corresponding controls.

\section{Immunofluorescent staining}

Immunofluorescent staining was carried out as described previously $[27,30,32]$. Briefly, cells were fixed with $4 \%$ methanol-free formaldehyde (Yeasen, \#36314ES76) for 20 min and permeabilized with $0.5 \%$ Triton X-100 for $20 \mathrm{~min}$ at $4{ }^{\circ} \mathrm{C}$. After rinsing with PBS for three times, cells were blocked for $1 \mathrm{~h}$ with $5 \%$ goat serum and incubated with anti-MORC2 (1:500), or antiOGT (1:500) antibody in $5 \%$ goat serum overnight at $4{ }^{\circ} \mathrm{C}$. Cells were rinsed with PBS three times and incubated with the secondary antibodies conjugated with Alexa 488 or Alexa-568 (1:500) at room temperature for 1 h. Then, cells were washed with PBS for three times, and sealed with a
DAPI-containing fluoroshield mounting medium (Abcam, \#ab104139). Images were visualized with Leica SP5 confocal microscope and analyzed.

\section{Purification of recombinant proteins}

The GST-tagged constructs in pGEX-6P-1 vector and His-tag constructs in pET-28a vector were transformed into $E$. coli strain BL21 (DE3) and incubated with $0.2 \mathrm{mM}$ IPTG (Invitrogen, \#15529019) to induce expression of recombination proteins at $16^{\circ} \mathrm{C}$ overnight. GST-tag proteins were purified using Glutathione Sepharose 4B beads (GE Healthcare, \#17075601), whereas His-tag proteins were purified using Ni-NTA agarose (TIANGEN Biotech, \#WM6-45-655-101), following the manufacturer's instructions. The purified proteins were immediately used for the experiments or frozen at $-80^{\circ} \mathrm{C}$.

\section{In vitro O-GIcNAcylation assays}

In vitro O-GIcNAcylation assays were performed as described previously [76]. Recombinant Flag-OGT protein purified from HEK293T cells and recombinant His-tagged MORC2 protein purified from E. coli were mixed in the reaction buffer $(50 \mathrm{mM}$ Tris- $\mathrm{HCl}$ pH 7.5, $12.5 \mathrm{mM} \mathrm{MgCl}, 2 \mathrm{mM}$ UDPGlcNAc, and $1 \mathrm{mM}$ DTT) in a final volume of $25 \mu \mathrm{l}$ per sample. The samples were incubated at $37^{\circ} \mathrm{C}$ for $24 \mathrm{~h}$. The reaction was resolved with SDS-PAGE, blotted onto a PVDF membrane, followed by immunoblotting with an antiO-GIcNAc antibody to detect O-GlcNAcylation of MORC2.

\section{qPCR and ChIP-qPCR}

Total RNA was isolated using TRlzol reagent (Invitrogen, \#15596018), and $1 \mu \mathrm{g}$ of RNA was subjected to CDNA synthesis using PrimeScript RT Master Mix (Takara, \#RR036). Quantitative real-time PCR (qPCR) was performed using SYBR Premix Ex Taq (Takara, \#RR420) following the manufacturer's instructions. The expression levels of the indicated mRNAs were calculated using the $2^{-\Delta \Delta C t}$ method and were normalized to internal control GAPDH. Chromatin immunoprecipitation (ChIP) assays were performed using SimpleChIP Enzymatic Chromatin IP Kit (magnetic beads) (Cell Signaling Technology, \#9003 S) according to the manufacturer's instructions. Quantitative results are displayed as corresponding fold change, and anti-rabbit lgG or anti-mouse lgG were used as a negative control. Primers used for QPCR and ChIP assays are listed in Supplementary Tables S7.

\section{Luciferase assays}

Cells were transfected with $200 \mathrm{ng}$ of pGL3, pGL3-SNAIL or pGL3-CTGF expression vectors using Lipofectamine 2000. Renilla luciferase expression vector (pRL) (5 ng) was also transfected into cells as a transfection control. After $48 \mathrm{~h}$ of transfection, luciferase assays were performed using a DualLuciferase Reporter Assay System (Promega, \#E1910) according to the manufacturer's instructions. The promoter activities were normalized to the corresponding values of Renilla luciferase.

\section{Transwell migration and invasion assays}

Transwell migration and invasion assays were performed as described previously [27] using Boyden chambers with $8 \mu \mathrm{m}$ pores (Corning Falcon, \#353097) and Matrigel Invasion Chambers (Corning BioCoat, \#354480), respectively. Medium containing 10\% FBS in the lower chamber served as a chemoattractant. The migrated and invaded cells at the bottom of the inserts were fixed with methanol for $30 \mathrm{~min}$ and stained with $0.1 \%$ crystal violet for $1 \mathrm{~h}$ at room temperature. Total number of cells in each chamber was counted. Cells were counted in 10 random fields under microscope.

\section{Lung metastasis assays}

All animal studies were approved by the Institutional Animal Care and Use Committee of Shanghai Cancer Center, Fudan University. For experimental metastasis assays, $2 \times 10^{6}$ MORC2-KO LM2-4175 cells stably expressing pMSCV or Flag-MORC2 (WT or T556A) in $200 \mu$ l of PBS were injected in the tail vein of six-week-old BALB/c female nude mice $(n=8$; Shanghai SLAC Laboratory Animal Co.). All mice were randomly divided into different groups. No statistical methods were used to estimate the number of mice. OSMI-1 (Sigma, \#SML1621) was given after one week of injection at a dose of $10 \mathrm{mg} / \mathrm{kg} / \mathrm{day}$ [77] (dissolved in corn oil with $0.05 \%$ DMSO) for four weeks, five days a week and two days off at weekends. Control groups were administrated with the same volume of vehicle control $(0.05 \%$ DMSO in corn oil). After five weeks of injection, the lungs were excised, fixed in Bouin's solution overnight, and metastatic lung nodules were counted under a Nikon SMZ1500 stereomicroscope. No blinding was performed during animal experiments. 


\section{Clinical samples and immunohistochemical staining}

All procedures were conducted in accordance with the Declaration of Helsinki and International Ethical Guidelines for Biomedical Research Involving Human Subjects and approved by the institutional ethics review board of Fudan University Shanghai Cancer Center. A total of 126 primary breast tumor specimens were obtained from breast cancer patients who underwent surgery at Fudan University Shanghai Cancer Center. These patients did not receive any therapies before surgical operation. Informed consent was obtained from all patients.

IHC staining was performed as described previously [78] using an antiOGT (Proteintech, \#11576, 1:200), anti-O-GIcNAc (ThermoFisher, \#MA1-072, 1: 200), anti-MORC2 (Novus, \#NBP1-89295, 1:150), anti-SNAIL (Abcam, \# ab224731, 1:200) or anti-CTGF (Proteintech, \#23936-1-AP, 1:200) antibody. The representative photographs were taken using Olympus BX43 microscope. Interpretation of the IHC results was performed by two independent pathologists who were blinded to the clinicopathological information. Slides were evaluated using light microscopy and a standard semiquantitative immunoreactivity score as described previously [78]. By recording the percentage of positive staining $(0,<5 \% ; 1,5-25 \% ; 2,26-50 \%$; $3,50-75 \%$; and $4,>75 \%)$ and staining intensity (0, negative; 1 , weak; 2 , moderate; and 3, strong) for each sample, immunoreactivity score (IRS) $(0-12)$ was calculated by multiplying positive staining percentage with staining intensity. A score of $0-4$ was considered as low expression and a score of 5-12 was considered as high expression.

\section{Statistical analysis}

All data are presented as mean \pm standard deviation from at least three independent experiments. The unpaired two-tailed Student's $t$ test was used to compare data between two groups using SPSS 20. The probability of survival was estimated with the Kaplan-Meier method and differences between groups were evaluated by the log-rank test. $P$ values of less than 0.05 were considered statistically significant.

\section{DATA AVAILABILITY}

All data generated or analyzed during this study are included in this article.

\section{REFERENCES}

1. Wang T, Yu Q, Li J, Hu B, Zhao Q, Ma C, et al. O-GlcNAcylation of fumarase maintains tumour growth under glucose deficiency. Nat Cell Biol. 2017:19:833-43.

2. Gloster TM, Vocadlo DJ. Mechanism, structure, and inhibition of O-GIcNAc processing enzymes. Curr Signal Transduct Ther. 2010;5:74-91.

3. Yang X, Qian K. Protein O-GlcNAcylation: emerging mechanisms and functions. Nat Rev Mol Cell Biol. 2017;18:452-65.

4. Chatham JC, Zhang J, Wende AR. Role of O-linked N-acetylglucosamine protein modification in cellular (patho)physiology. Physiol Rev. 2021;101:427-93.

5. Zhang N, Zhu T, Yu K, Shi M, Wang X, Wang L, et al. Elevation of O-GlcNAc and GFAT expression by nicotine exposure promotes epithelial-mesenchymal transition and invasion in breast cancer cells. Cell Death Dis. 2019;10:343.

6. Wang ZV, Deng Y, Gao N, Pedrozo Z, Li DL, Morales CR, et al. Spliced X-box binding protein 1 couples the unfolded protein response to hexosamine biosynthetic pathway. Cell. 2014;156:1179-92.

7. Manzari B, Kudlow JE, Fardin P, Merello E, Ottaviano C, Puppo M, et al. Induction of macrophage glutamine: fructose-6-phosphate amidotransferase expression by hypoxia and by picolinic acid. Int J Immunopathol Pharm. 2007;20:47-58.

8. Paterson AJ, Kudlow JE. Regulation of glutamine:fructose-6-phosphate amidotransferase gene transcription by epidermal growth factor and glucose. Endocrinology. 1995;136:2809-16.

9. Shimizu M, Tanaka N. IL-8-induced O-GICNAc modification via GLUT3 and GFAT regulates cancer stem cell-like properties in colon and lung cancer cells. Oncogene. 2019;38:1520-33.

10. Eguchi $\mathrm{S}$, Oshiro $\mathrm{N}$, Miyamoto $\mathrm{T}$, Yoshino $\mathrm{K}$, Okamoto $\mathrm{S}$, Ono $\mathrm{T}$, et al. AMPactivated protein kinase phosphorylates glutamine: fructose-6-phosphate amidotransferase 1 at Ser243 to modulate its enzymatic activity. Genes Cells. 2009;14:179-89.

11. Li Y, Roux C, Lazereg S, LeCaer JP, Laprevote O, Badet B, et al. Identification of a novel serine phosphorylation site in human glutamine:fructose-6-phosphate amidotransferase isoform 1. Biochemistry. 2007;46:13163-9.

12. Chang Q, Su K, Baker JR, Yang X, Paterson AJ, Kudlow JE. Phosphorylation of human glutamine:fructose-6-phosphate amidotransferase by cAMP-dependent protein kinase at serine 205 blocks the enzyme activity. J Biol Chem. 2000;275:21981-7.
13. Marshall S, Bacote V, Traxinger RR. Complete inhibition of glucose-induced desensitization of the glucose transport system by inhibitors of mRNA synthesis. Evidence for rapid turnover of glutamine:fructose-6-phosphate amidotransferase. J Biol Chem. 1991;266:10155-61.

14. Slawson C, Hart GW. O-GlcNAc signalling: implications for cancer cell biology. Nat Rev Cancer. 2011;11:678-84.

15. Walter LA, Lin YH, Halbrook CJ, Chuh KN, He L, Pedowitz NJ, et al. Inhibiting the hexosamine biosynthetic pathway lowers O-GIcNAcylation levels and sensitizes cancer to environmental stress. Biochemistry. 2020;59:3169-79.

16. Asthana A, Ramakrishnan P, Vicioso Y, Zhang K, Parameswaran R. Hexosamine biosynthetic pathway inhibition leads to AML cell differentiation and cell death. Mol Cancer Ther. 2018;17:2226-37.

17. Kumar R, Li DQ, Muller S, Knapp S. Epigenomic regulation of oncogenesis by chromatin remodeling. Oncogene. 2016;35:4423-36.

18. Li DQ, Nair SS, Kumar R. The MORC family: new epigenetic regulators of transcription and DNA damage response. Epigenetics. 2013;8:685-93.

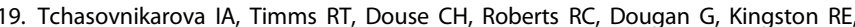
et al. Hyperactivation of HUSH complex function by Charcot-Marie-Tooth disease mutation in MORC2. Nat Genet. 2017;49:1035-44.

20. Douse CH, Bloor S, Liu Y, Shamin M, Tchasovnikarova IA, Timms RT, et al. Neuropathic MORC2 mutations perturb GHKL ATPase dimerization dynamics and epigenetic silencing by multiple structural mechanisms. Nat Commun. 2018;9:651.

21. Li DQ, Nair SS, Ohshiro K, Kumar A, Nair VS, Pakala SB, et al. MORC2 signaling integrates phosphorylation-dependent, ATPase-coupled chromatin remodeling during the DNA damage response. Cell Rep. 2012;2:1657-69.

22. Liu N, Lee CH, Swigut T, Grow E, Gu B, Bassik MC, et al. Selective silencing of euchromatic L1s revealed by genome-wide screens for L1 regulators. Nature. 2018;553:228-32.

23. Sevilla T, Lupo V, Martinez-Rubio D, Sancho $P$, Sivera $R$, Chumillas MJ, et al. Mutations in the MORC2 gene cause axonal Charcot-Marie-Tooth disease. Brain: a J Neurol. 2016;139:62-72.

24. Albulym OM, Kennerson ML, Harms MB, Drew AP, Siddell AH, Auer-Grumbach $M$, et al. MORC2 mutations cause axonal Charcot-Marie-Tooth disease with pyramidal signs. Ann Neurol. 2016;79:419-27.

25. Ando M, Okamoto Y, Yoshimura A, Yuan JH, Hiramatsu Y, Higuchi Y, et al. Clinical and mutational spectrum of Charcot-Marie-Tooth disease type $2 Z$ caused by MORC2 variants in Japan. Eur J Neurol. 2017;24:1274-82.

26. Guillen Sacoto MJ, Tchasovnikarova IA, Torti E, Forster C, Andrew EH, Anselm I, et al. De Novo Variants in the ATPase Module of MORC2 Cause a Neurodevelopmental Disorder with Growth Retardation and Variable Craniofacial Dysmorphism. Am J Hum Genet. 2020;107:352-63.

27. Zhang FL, Cao JL, Xie HY, Sun R, Yang LF, Shao ZM, et al. Cancer-associated MORC2-mutant M276I regulates an hnRNPM-mediated CD44 splicing switch to promote invasion and metastasis in triple-negative breast cancer. Cancer Res. 2018;78:5780-92.

28. Ding QS, Zhang L, Wang BC, Zeng Z, Zou XQ, Cao PB, et al. Aberrant high expression level of MORC2 is a common character in multiple cancers. Hum Pathol. 2018;76:58-67.

29. Pan Z, Ding Q, Guo Q, Guo Y, Wu L, Wu L, et al. MORC2, a novel oncogene, is upregulated in liver cancer and contributes to proliferation, metastasis and chemoresistance. Int J Oncol. 2018;53:59-72.

30. Liu HY, Liu YY, Yang F, Zhang L, Zhang FL, Hu X, et al. Acetylation of MORC2 by NAT10 regulates cell-cycle checkpoint control and resistance to DNA-damaging chemotherapy and radiotherapy in breast cancer. Nucleic Acids Res. 2020;48:3638-56.

31. Yang F, Xie HY, Yang LF, Zhang L, Zhang FL, Liu HY, et al. Stabilization of MORC2 by estrogen and antiestrogens through GPER1- PRKACA-CMA pathway contributes to estrogen-induced proliferation and endocrine resistance of breast cancer cells. Autophagy. 2020;16:1061-76.

32. Zhang L, Li DQ. MORC2 regulates DNA damage response through a PARP1dependent pathway. Nucleic Acids Res. 2019;47:8502-20.

33. Martinez-Fleites C, Macauley MS, He Y, Shen DL, Vocadlo DJ, Davies GJ. Structure of an O-GlcNAc transferase homolog provides insight into intracellular glycosylation. Nat Struct Mol Biol. 2008;15:764-5.

34. Ortiz-Meoz RF, Jiang J, Lazarus MB, Orman M, Janetzko J, Fan C, et al. A small molecule that inhibits OGT activity in cells. ACS Chem Biol. 2015;10:1392-7.

35. Tao T, He Z, Shao Z, Lu H. TAB3 O-GlcNAcylation promotes metastasis of triple negative breast cancer. Oncotarget. 2016;7:22807-18.

36. Hart GW, Housley MP, Slawson C. Cycling of O-linked beta- $\mathrm{N}$-acetylglucosamine on nucleocytoplasmic proteins. Nature. 2007;446:1017-22.

37. Macauley MS, Vocadlo DJ. Increasing O-GIcNAc levels: An overview of smallmolecule inhibitors of O-GIcNAcase. Biochim Biophys Acta. 2010;1800:107-21.

38. Shalem O, Sanjana NE, Hartenian E, Shi X, Scott DA, Mikkelson T, et al. Genomescale CRISPR-Cas9 knockout screening in human cells. Science. 2014;343:84-87.

39. Inman GJ, Nicolas FJ, Callahan JF, Harling JD, Gaster LM, Reith AD, et al. SB431542 is a potent and specific inhibitor of transforming growth factor-beta 
superfamily type I activin receptor-like kinase (ALK) receptors ALK4, ALK5, and ALK7. Mol Pharm. 2002;62:65-74.

40. Lucena MC, Carvalho-Cruz P, Donadio JL, Oliveira IA, de Queiroz RM, MarinhoCarvalho MM, et al. Epithelial mesenchymal transition induces aberrant glycosylation through hexosamine biosynthetic pathway activation. J Biol Chem. 2016:291:12917-29.

41. Muraoka RS, Dumont N, Ritter CA, Dugger TC, Brantley DM, Chen J, et al. Blockade of TGF-beta inhibits mammary tumor cell viability, migration, and metastases. J Clin Invest. 2002;109:1551-9.

42. Fang $Y$, Chen $Y, Y u$ L, Zheng C, Qi Y, Li Z, et al. Inhibition of breast cancer metastases by a novel inhibitor of TGFbeta receptor 1. J Natl Cancer Inst. 2013;105:47-58.

43. Son JY, Park SY, Kim SJ, Lee SJ, Park SA, Kim MJ, et al. EW-7197, a novel ALK-5 kinase inhibitor, potently inhibits breast to lung metastasis. Mol Cancer Ther. 2014;13:1704-16.

44. Derynck R, Zhang YE. Smad-dependent and Smad-independent pathways in TGFbeta family signalling. Nature. 2003;425:577-84.

45. Shao P, Liu Q, Maina PK, Cui J, Bair TB, Li T, et al. Histone demethylase PHF8 promotes epithelial to mesenchymal transition and breast tumorigenesis. Nucleic Acids Res. 2017;45:1687-702.

46. Zhou T, Che D, Lan Y, Fang Z, Xie J, Gong H, et al. Mesenchymal marker expression is elevated in Muller cells exposed to high glucose and in animal models of diabetic retinopathy. Oncotarget. 2017;8:4582-94.

47. Sonnylal S, Xu S, Jones H, Tam A, Sreeram VR, Ponticos M, et al. Connective tissue growth factor causes EMT-like cell fate changes in vivo and in vitro. J Cell Sci. 2013;126:2164-75.

48. Lee SW, Won JY, Kim WJ, Lee J, Kim KH, Youn SW, et al. Snail as a potential target molecule in cardiac fibrosis: paracrine action of endothelial cells on fibroblasts through snail and CTGF axis. Mol Ther. 2013;21:1767-77.

49. Che D, Zhou T, Lan Y, Xie J, Gong H, Li C, et al. High glucose-induced epithelialmesenchymal transition contributes to the upregulation of fibrogenic factors in retinal pigment epithelial cells. Int J Mol Med. 2016;38:1815-22.

50. Copeland RJ, Han G, Hart GW. O-GIcNAcomics-Revealing roles of O-GIcNAcylation in disease mechanisms and development of potential diagnostics. Proteom Clin Appl. 2013;7:597-606.

51. Wang T, Qin ZY, Wen LZ, Guo Y, Liu Q, Lei ZJ, et al. Epigenetic restriction of Hippo signaling by MORC2 underlies stemness of hepatocellular carcinoma cells. Cell Death Differ. 2018;25:2086-2100.

52. Cameron A, Giacomozzi B, Joyce J, Gray A, Graham D, Ousson S, et al. Generation and characterization of a rabbit monoclonal antibody site-specific for tau O-GlcNAcylated at serine 400. FEBS Lett. 2013;587:3722-8

53. Shan H, Sun J, Shi M, Liu X, Shi Z, Yu W, et al. Generation and characterization of a site-specific antibody for SIRT1 O-GIcNAcylated at serine 549. Glycobiology. 2018;28:482-7.

54. Pathak S, Borodkin VS, Albarbarawi O, Campbell DG, Ibrahim A, van Aalten DM. O-GlcNAcylation of TAB1 modulates TAK1-mediated cytokine release. EMBO J. 2012;31:1394-404.

55. Kamemura K, Hayes BK, Comer Fl, Hart GW. Dynamic interplay between O-glycosylation and O-phosphorylation of nucleocytoplasmic proteins: alternative glycosylation/ phosphorylation of THR-58, a known mutational hot spot of c-Myc in lymphomas, is regulated by mitogens. J Biol Chem. 2002;277:19229-35.

56. Klein AL, Berkaw MN, Buse MG, Ball LE. O-linked N-acetylglucosamine modification of insulin receptor substrate- 1 occurs in close proximity to multiple $\mathrm{SH} 2$ domain binding motifs. Mol Cell Proteom. 2009;8:2733-45.

57. Hanover JA, Krause MW, Love DC. The hexosamine signaling pathway: O-GlcNAC cycling in feast or famine. Biochim Biophys Acta. 2010;1800:80-95.

58. Oikari S, Kettunen T, Tiainen S, Häyrinen J, Masarwah A, Sudah M, et al. UDP-sugar accumulation drives hyaluronan synthesis in breast cancer. Matrix Biol. 2018;67:63-74.

59. Vasconcelos-Dos-Santos A, Loponte HF, Mantuano NR, Oliveira IA, de Paula IF, Teixeira LK, et al. Hyperglycemia exacerbates colon cancer malignancy through hexosamine biosynthetic pathway. Oncogenesis. 2017;6:e306.

60. Chen W, Do KC, Saxton B, Leng S, Filipczak P, Tessema M, et al. Inhibition of the hexosamine biosynthesis pathway potentiates cisplatin cytotoxicity by decreasing BiP expression in non-small-cell lung cancer cells. Mol Carcinog. 2019;58:1046-55.

61. Dong T, Kang X, Liu Z, Zhao S, Ma W, Xuan Q, et al. Altered glycometabolism affects both clinical features and prognosis of triple-negative and neoadjuvant chemotherapy-treated breast cancer. Tumour Biol. 2016;37:8159-68.

62. Guillaumond F, Leca J, Olivares O, Lavaut MN, Vidal N, Berthezene P, et al. Strengthened glycolysis under hypoxia supports tumor symbiosis and hexosamine biosynthesis in pancreatic adenocarcinoma. Proc Natl Acad Sci USA. 2013;110:3919-24.

63. Li L, Shao M, Peng P, Yang C, Song S, Duan F, et al. High expression of GFAT1 predicts unfavorable prognosis in patients with hepatocellular carcinoma. Oncotarget. 2017;8:19205-17.
64. Sharma NS, Gupta VK, Garrido VT, Hadad R, Durden BC, Kesh K, et al. Targeting tumor-intrinsic hexosamine biosynthesis sensitizes pancreatic cancer to anti-PD1 therapy. J Clin Invest. 2020;130:451-65.

65. Weigert C, Brodbeck K, Lehmann R, Haring HU, Schleicher ED. Overexpression of glutamine:fructose-6-phosphate-amidotransferase induces transforming growth factor-beta1 synthesis in NIH-3T3 fibroblasts. FEBS Lett. 2001;488:95-99.

66. Weigert C, Friess U, Brodbeck K, Häring HU, Schleicher ED. Glutamine:fructose-6phosphate aminotransferase enzyme activity is necessary for the induction of TGF-beta1 and fibronectin expression in mesangial cells. Diabetologia. 2003;46:852-5.

67. Peinado H, Olmeda D, Cano A. Snail, Zeb and bHLH factors in tumour progression: an alliance against the epithelial phenotype? Nat Rev Cancer. 2007;7:415-28.

68. Kang Y, Siegel PM, Shu W, Drobnjak M, Kakonen SM, Cordón-Cardo C, et al. A multigenic program mediating breast cancer metastasis to bone. Cancer Cell. 2003;3:537-49.

69. Batlle E, Sancho E, Franci C, Dominguez D, Monfar M, Baulida J, et al. The transcription factor snail is a repressor of E-cadherin gene expression in epithelial tumour cells. Nat Cell Biol. 2000;2:84-89.

70. Cano A, Perez-Moreno MA, Rodrigo I, Locascio A, Blanco MJ, del Barrio MG, et al. The transcription factor snail controls epithelial-mesenchymal transitions by repressing E-cadherin expression. Nat Cell Biol. 2000;2:76-83.

71. Moody SE, Perez D, Pan TC, Sarkisian CJ, Portocarrero CP, Sterner CJ, et al. The transcriptional repressor Snail promotes mammary tumor recurrence. Cancer Cell. 2005;8:197-209.

72. Tran HD, Luitel K, Kim M, Zhang K, Longmore GD, Tran DD. Transient SNAIL1 expression is necessary for metastatic competence in breast cancer. Cancer Res. 2014;74:6330-40.

73. Chen PS, Wang MY, Wu SN, Su JL, Hong CC, Chuang SE, et al. CTGF enhances the motility of breast cancer cells via an integrin-alphavbeta3-ERK1/2-dependent S100A4-upregulated pathway. J Cell Sci. 2007;120:2053-65.

74. Chien W, O'Kelly J, Lu D, Leiter A, Sohn J, Yin D, et al. Expression of connective tissue growth factor (CTGF/CCN2) in breast cancer cells is associated with increased migration and angiogenesis. Int J Oncol. 2011;38:1741-7.

75. Xie D, Nakachi K, Wang $H$, Elashoff $R$, Koeffler HP. Elevated levels of connective tissue growth factor, WISP-1, and CYR61 in primary breast cancers associated with more advanced features. Cancer Res. 2001;61:8917-23.

76. Pyo KE, Kim CR, Lee M, Kim JS, Kim Kl, Baek SH. ULK1 O-GlcNAcylation is crucial for activating VPS34 via ATG14L during autophagy initiation. Cell Rep. 2018;25:2878-90 e2874.

77. Sharma NS, Gupta VK, Dauer P, Kesh K, Hadad R, Giri B, et al. O-GlcNAc modification of Sox2 regulates self-renewal in pancreatic cancer by promoting its stability. Theranostics. 2019;9:3410-24.

78. Wang Q, Li M, Gan Y, Jiang S, Qiao J, Zhang W, et al. Mitochondrial protein UQCRC1 is oncogenic and a potential therapeutic target for pancreatic cancer. Theranostics. 2020;10:2141-57.

\section{ACKNOWLEDGEMENTS}

We sincerely thank Prof. Hao-Jie Lu and his lab members (Institutes of Biomedical Sciences, Fudan University) for technical assistance in electron transfer dissociationmass spectrometry (ETD-MS) analysis for identifying MORC2 O-GlcNAcylation sites. We are grateful to members of the Li laboratory for technical assistance and insightful discussions. This work was supported, in whole or in part, by National Natural Science Foundation of China (No. 81772805, 81972461, and 82173275) and the National Key R\&D Program of China (No. 2017YFC0908400 and 2018YFE0201600) to DQL.

\section{AUTHOR CONTRIBUTIONS}

YYL and HYL performed the experiments. TJY and FLZ contributed to data analysis. QL carried out experiments during the early stage of this project. GYL, ZMS, and DQL conceived the project and supervised the study. LYY and DQL wrote the paper with inputs from all authors.

\section{COMPETING INTERESTS}

The authors declare no competing interests.

\section{ETHICS APPROVAL}

This study was approved by the institutional ethics review board of Fudan University Shanghai Cancer Center and by the Institutional Animal Care and Use Committee of Fudan University Shanghai Cancer Center. 


\section{ADDITIONAL INFORMATION}

Supplementary information The online version contains supplementary material available at https://doi.org/10.1038/s41418-021-00901-0.

Correspondence and requests for materials should be addressed to Guang-Yu Liu Zhi-Ming Shao or Da-Qiang Li.

Reprints and permission information is available at http://www.nature.com/ reprints

Publisher's note Springer Nature remains neutral with regard to jurisdictional claims in published maps and institutional affiliations.
Open Access This article is licensed under a Creative Commons Attribution 4.0 International License, which permits use, sharing, adaptation, distribution and reproduction in any medium or format, as long as you give appropriate credit to the original author(s) and the source, provide a link to the Creative Commons license, and indicate if changes were made. The images or other third party material in this article are included in the article's Creative Commons license, unless indicated otherwise in a credit line to the material. If material is not included in the article's Creative Commons license and your intended use is not permitted by statutory regulation or exceeds the permitted use, you will need to obtain permission directly from the copyright holder. To view a copy of this license, visit http://creativecommons. org/licenses/by/4.0/.

(c) The Author(s) 2021 